\title{
Incremental-secant modulus iteration scheme and stress recovery for simulating cracking process in quasi-brittle materials using XFEM
}

\author{
Q. Z. Xiao ${ }^{1}$, B. L. Karihaloo ${ }^{1, *, \dagger}$ and X. Y. Liu ${ }^{2}$ \\ ${ }^{1}$ School of Engineering, Cardiff University, Queen's Buildings, The Parade, Newport Road, \\ Cardiff CF24 3AA, U.K. \\ ${ }^{2}$ Division of Engineering Sciences, Institute of Mechanics, Chinese Academy of Sciences, \\ No.15 Beisihuanxi Road, Beijing 100080, China
}

\begin{abstract}
SUMMARY
In this paper, an incremental-secant modulus iteration scheme using the extended/generalized finite element method (XFEM) is proposed for the simulation of cracking process in quasi-brittle materials described by cohesive crack models whose softening law is composed of linear segments. The leading term of the displacement asymptotic field at the tip of a cohesive crack (which ensures a displacement discontinuity normal to the cohesive crack face) is used as the enrichment function in the XFEM. The opening component of the same field is also used as the initial guess opening profile of a newly extended cohesive segment in the simulation of cohesive crack propagation. A statically admissible stress recovery (SAR) technique is extended to cohesive cracks with special treatment of non-homogeneous boundary tractions. The application of locally normalized co-ordinates to eliminate possible ill-conditioning of SAR, and the influence of different weight functions on SAR are also studied. Several mode I cracking problems in quasi-brittle materials with linear and bilinear softening laws are analysed to demonstrate the usefulness of the proposed scheme, as well as the characteristics of global responses and local fields obtained numerically by the XFEM. Copyright (C) 2006 John Wiley \& Sons, Ltd.
\end{abstract}

Received 24 February 2006; Revised 27 June 2006; Accepted 13 July 2006

KEY WORDS: cohesive crack; extended/generalized finite element method (XFEM); quasi-brittle materials; secant modulus iteration; statically admissible stress recovery (SAR)

\footnotetext{
*Correspondence to: B. L. Karihaloo, School of Engineering, Cardiff University, Queen's Buildings, Newport Road, Cardiff CF24 3AA, U.K.

${ }^{\dagger}$ E-mail: karihaloob@ cardiff.ac.uk

Contract/grant sponsor: European Commission KMM-Network of Excellence
}

Copyright (C) 2006 John Wiley \& Sons, Ltd. 


\section{INTRODUCTION}

The extended/generalized finite element method (XFEM) (Moës et al. [1], Strouboulis et al. [2], Karihaloo and Xiao [3], Babuška et al. [4], Xiao and Karihaloo [5]) is promising for the simulation of cracking or failure process in materials and structures. It enriches the standard local FE approximations with known information about the problem, such as a displacement discontinuity across a crack, the asymptotic solution at a crack tip, or a strain discontinuity across an interface, with the use of the partition of unity (PU) (Babuska and Melenk [6]). Therefore, in contrast with the FEM, it avoids the use of meshes conforming with the discontinuity and also adaptive remeshing as the discontinuity grows.

Cohesive zone (or crack) models (Dugdale [7], Barenblatt [8], Hillerborg et al. [9]) have been extensively used in the study of localization and failure in engineering materials and structures. Elices et al. [10] have discussed the advantages and limitations of these models. In the most widely used standard formulation of the cohesive crack model for quasi-brittle materials (see Figure 1), it is assumed that the stress-strain behaviour is isotropic linear elastic, and that the crack is initiated at a point where the maximum tensile principal stress $\sigma_{1}$ reaches the tensile strength $f_{t}$ of the uncracked material, and that the crack is oriented normal to the direction of $\sigma_{1}$. An evolution law is also postulated for the monotonic mode I loading so that the cohesive stress is a unique function of the crack opening which, for concrete, decreases monotonically along the cohesive zone. The cohesive crack propagates when the maximum tensile principal stress $\sigma_{1}$ at its tip reaches $f_{t}$. Although this standard formulation of the cohesive crack model is highly simplified, it is able to capture the essence of the fracture process in concrete specimens and structures (see, e.g. Karihaloo [11]).

Several researchers have studied cohesive crack propagation in quasi-brittle materials using the XFEM. Wells and Sluys [12] and Moës and Belytschko [13] analysed a continuous cohesive crack that runs through an existing FE mesh. Remmers et al. [14] studied the possibility of defining cohesive segments that can arise at arbitrary locations and in arbitrary directions and thus allow for the resolution of complex crack patterns including crack nucleation at multiple locations, followed by growth and coalescence. de Borst et al. [15] have given a concise overview of the various ways to numerically implement the cohesive zone methodology. They concluded that the XFEM provides a proper representation of the discrete character of cohesive zone formulations avoiding any mesh bias.

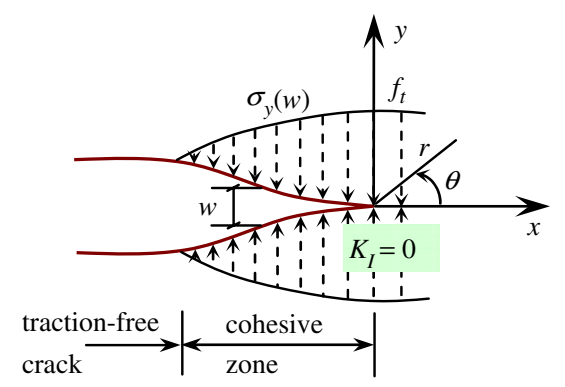

Figure 1. A real traction-free crack terminating in a fracture process (cohesive) zone (FPZ) with residual stress transfer capacity $\sigma_{y}(w)$ whose faces close smoothly near its tip $\left(K_{I}=0\right)$. The material outside the

FPZ is linear elastic, but within the FPZ is softening. 
In the XFEM formulation of the cohesive zone model, Wells and Sluys [12] used the jump function as an enrichment function for the whole cohesive crack, hence the cohesive crack tip touches the element boundary. Moës and Belytschko [13] used the jump function for the part of the cohesive crack not adjacent to its tip, and a branch function adjacent to the tip. This approach can handle cases in which the cohesive crack tip does not touch the element boundary. However, the adopted branch function does not represent the true asymptotic nature of the displacement/stress field adjacent to the cohesive crack tip. Hansbo and Hansbo [16] modelled strong and weak discontinuities in solid mechanics by considering an element traversed by a discontinuity as a double element each half of which is used for the interpolation of one side of the discontinuity. This approach is difficult to use when the discontinuity ends within an element. Recently, Areias and Belytschko [17] discussed the relationship between this approach and the XFEM. Zi and Belytschko [18] enriched all cracked linear 3-node or quadratic 6-node triangular elements including the elements containing the crack tip by the sign function. Alfaiate et al. [19] embedded displacement jumps which do not need to be homogeneous within each FE. Mariani and Perego [20] introduced in a standard FE model a cubic displacement discontinuity, in order to reproduce the typical cusplike shape of the process zone at the tip of a cohesive crack. However, this cubic function does not represent the true angular distribution of the displacement adjacent to the tip.

Rubinstein [21] has shown that relatively small errors in the determination of the crack path deflection angle can lead to a significant cumulative deviation of the crack path over a finite crack length. Therefore, a reliable analysis of cohesive crack propagation requires not only a suitable criterion of crack growth but also an accurate knowledge of the crack tip field. Recently, Xiao and Karihaloo [22] have demonstrated that, for a crack with traction-free faces, when the crack tip asymptotic field is available and used as enrichment function, the XFEM not only avoids the use of a mesh conforming with the crack but it is also more accurate than the FEM. However, it is necessary to ensure that the unknown coefficients of the crack tip field at the enriched nodes are all equal to one another. Hence the XFEM can use a much coarser mesh around the crack tip. However, when the enrichment function does not represent the true asymptotic nature of the crack tip field, the mesh needs to be refined in the same manner as in the FEM. Thus it is advantageous to use the true asymptotic fields around a cohesive crack tip in the XFEM.

In this paper, we will use the leading term of the displacement asymptotic field at the tip of a cohesive crack in quasi-brittle materials obtained recently by Xiao and Karihaloo [23] as the crack tip enrichment function for the simulation of cracking or failure process using the XFEM. In the published literature (see, e.g. [12, 18-20]), incremental-tangent modulus iteration schemes are frequently adopted. For many widely used cohesive crack models for quasi-brittle materials, the softening law is composed of linear segments, e.g. linear and bilinear softening laws. It seems possible to use the simpler incremental-secant modulus iteration strategy. This strategy has been used by Moës and Belytschko [13] for a linear softening law, and by Karihaloo et al. [24] for a bilinear softening law in the semi-analytical analysis of the cohesive zone in quasi-brittle materials. The application of this strategy to non-linear softening laws composed of linear segments within the XFEM will be detailed and demonstrated in this study.

In order to determine more accurately the direction of propagation of the cohesive crack tip, Wells and Sluys [12] used non-local stresses at the tip to find the principal directions. These stresses were calculated as a weighted average of stresses using a Gaussian weight function

$$
w(r)=\frac{1}{(2 \pi)^{3 / 2} l^{3}} \exp \left(-\frac{r^{2}}{2 l^{2}}\right)
$$


where $w(r)$ is the weight, $l$ determines how quickly the weight function decays away from the tip and is taken as approximately three times the typical element size, and $r$ is the distance of a point from the tip. At the end of a load increment, if the maximum principal tensile stress at all integration points in the element ahead of the crack exceeds $f_{t}$, a discontinuity is introduced through the entire element. For each in-plane stress component, Mariani and Perego [20] fitted a complete fourth-order polynomial to the stress values at the Gauss points belonging to a semi-circle ahead of the process zone tip, with a radius up to eight times the element characteristic dimension using the least-squares optimization. These stress recovery techniques have not paid attention to the angular distribution of the stress adjacent to the crack tip. For this purpose, the statically admissible stress recovery (SAR) scheme of Xiao and Karihaloo [22,25], which has been shown to be very powerful for the traditional FEM as well as the XFEM in linear elastic problems with traction-free boundary segments, will be extended to cohesive cracks. SAR uses basis functions, which meet the equilibrium equations within the domain and the local traction conditions on the boundary, and moving least squares (MLS) to fit the stresses at sampling points (e.g. quadrature points) obtained by the XFEM. Techniques for handling non-homogeneous tractions on the boundary will be discussed. Furthermore, the normalization of local co-ordinates suggested by Zienkiewicz et al. [26] will be employed to eliminate the possible ill-conditioning of MLS. The influence of different weight functions on the recovered stresses of SAR will also be studied. The most widely used stress recovery scheme (denoted as AVG) will also be used for comparison. AVG simply averages the stress values at each node evaluated from adjacent elements by bi-linear extrapolation from the Gauss points, and interpolates the averaged stresses using shape functions.

Finally, the cracking or failure process of several typical quasi-brittle material specimens with linear and bilinear softening laws will be analysed to demonstrate the usefulness of the proposed scheme, as well as the characteristics of global responses and local fields obtained numerically by the XFEM.

\section{INCREMENTAL-SECANT MODULUS ITERATION SCHEME FOR COHESIVE CRACK MODELLING OF QUASI-BRITTLE MATERIALS USING XFEM}

To model the cohesive cracks in the XFEM, a standard local FE displacement approximation around the crack is enriched with discontinuous Heaviside functions along the crack faces behind the crack tip including the open traction-free part, and the crack tip asymptotic displacement fields at nodes surrounding the cohesive crack tip using the PU. The approximation of displacements for an element can be expressed in the following form:

$$
\left\{\begin{array}{l}
u^{h}(x) \\
v^{h}(x)
\end{array}\right\}=\sum_{i \in I} \phi_{i}(x)\left\{\begin{array}{c}
u_{0 i} \\
v_{0 i}
\end{array}\right\}+\sum_{j \in J \cap I} \phi_{j}(x) H(x)\left\{\begin{array}{l}
b_{1 j} \\
b_{2 j}
\end{array}\right\}+\sum_{m \in M_{k} \cap I} \phi_{m}(x)\left\{\begin{array}{l}
u_{m}^{(\mathrm{tip} k)} \\
v_{m}^{(\mathrm{tip} k)}
\end{array}\right\}
$$

where $I$ is the set of all nodes in the element, $\left(u_{0 i}, v_{0 i}\right)$ are the regular degrees of freedom at node $i, \phi_{i}$ is the FE shape function associated with node $i, J$ is the subset of nodes whose support is intersected by the crack but do not cover any cohesive crack tips, the function $H(x)$ is the Heaviside function centred on the crack discontinuity, and $\left(b_{1 j}, b_{2 j}\right)$ are the corresponding additional degrees of freedom. $M_{k}$ is the subset of nodes that are enriched around the cohesive crack tip $k$ with the asymptotic displacements $u^{(\operatorname{tip} k)}$ and $v^{(\operatorname{tip} k)} \cdot u_{m}^{(\operatorname{tip} k)}$ and $v_{m}^{(\operatorname{tip} k)}$ are enrichment functions adopted at node $m$ with corresponding nodal parameters independent of the other nodes. 


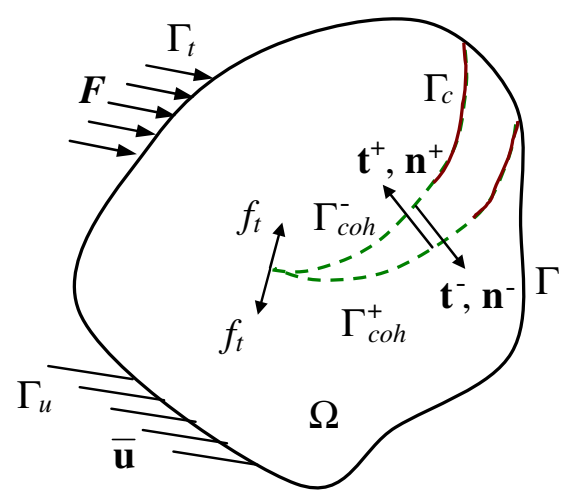

Figure 2. Body with a crack $\Gamma_{c}$ involving a cohesive zone $\Gamma_{\text {coh }}$ subjected to prescribed boundary tractions $\mathbf{F}$ on $\Gamma_{t}$ and displacements $\overline{\mathbf{u}}$ on $\Gamma_{u}$.

Consider a domain $\Omega$ containing a crack $\Gamma_{c}$, as shown in Figure 2. The boundary $\Gamma$ is composed of the segments $\Gamma_{u}, \Gamma_{t}$, and $\Gamma_{c}$. The part of the crack on which a softening law is active, i.e. the FPZ, is denoted by $\Gamma_{\text {coh. }}$. Prescribed displacements $\overline{\mathbf{u}}$ are imposed on $\Gamma_{u}$, while tractions $\mathbf{F}$ are imposed on $\Gamma_{t}$. The crack surface $\Gamma_{c}$ excluding $\Gamma_{\text {coh }}$ is assumed to be traction-free. The cohesive tractions $\mathbf{t}^{+}, \mathbf{t}^{-}$are imposed on the upper and lower surfaces of $\Gamma_{\text {coh. }}$.

The virtual work equation without body forces is given by $[12,13]$

$$
\int_{\Omega} \boldsymbol{\sigma}(\mathbf{u}): \nabla^{s} \mathbf{v} \mathrm{d} \Omega+\int_{\Gamma_{\text {coh }}} \mathbf{t} \cdot \mathbf{w}(\mathbf{v}) \mathrm{d} \Gamma=\int_{\Gamma_{t}} \mathbf{F} \cdot \mathbf{v} \mathrm{d} \Gamma
$$

where $\boldsymbol{\sigma}$ is the Cauchy stress, and $\nabla^{s}$ denotes the symmetric part of the gradient operator. The displacements $\mathbf{u}$ must belong to the space $U$ of kinematically admissible displacement fields

$$
\mathbf{u} \in U=\left\{\mathbf{u} \in U: \mathbf{u}=\overline{\mathbf{u}} \text { on } \Gamma_{u}, \mathbf{u} \text { discontinuous on } \Gamma_{c}\right\}
$$

where the space $U$ is related to the regularity of the solution. The test function $\mathbf{v}$ must belong to the space $V$ defined by

$$
\mathbf{v} \in V=\left\{\mathbf{v} \in V: \mathbf{v}=0 \text { on } \Gamma_{u}, \mathbf{v} \text { discontinuous on } \Gamma_{c}\right\}
$$

The cohesive tractions $\mathbf{t}=\mathbf{t}^{+}=-\mathbf{t}^{-}$and the separation $\mathbf{w}(\mathbf{v})=\mathbf{v}^{-}-\mathbf{v}^{+}$are related by a softening law on $\Gamma_{\text {coh. }}$.

For a softening law composed of $n$ linear segments, the relationship between the normal cohesive stress and opening for the $i$ th segment $(i=1,2, \ldots, n)$ can be expressed as

$$
\sigma_{y}=f_{i}-k_{i} w
$$

where $\boldsymbol{\sigma}_{y}=\mathbf{t} \cdot \mathbf{n}$ is the normal traction and $w(v)=\mathbf{w}(\mathbf{v}) \cdot \mathbf{n}$ is the normal separation. The unit normal is $\mathbf{n}=\mathbf{n}^{+}=-\mathbf{n}^{-}$, and $\mathbf{n}^{+}, \mathbf{n}^{-}$are the normals to the upper and lower surfaces of $\Gamma_{\text {coh }}$, respectively. ' $-k_{i}$ ' is the slope of the $i$ th segment and ' $f_{i}$ ' gives the $\sigma_{y}$-intercept. For simplicity but 
without loss of generality, the relationship (5) is used for both loading ( $w$ increases) and unloading ( $w$ decreases) stages. The virtual work Equation (2) can thus be simplified to read

$$
\int_{\Omega} \mathbf{D} \boldsymbol{\varepsilon}(\mathbf{u}): \boldsymbol{\varepsilon}(\mathbf{v}) \mathrm{d} \Omega-\int_{\Gamma_{\mathrm{coh}}} k_{i} w(\mathbf{u}) w(\mathbf{v}) \mathrm{d} \Gamma=\int_{\Gamma_{t}} \mathbf{F} \cdot \mathbf{v} \mathrm{d} \Gamma-\int_{\Gamma_{\mathrm{coh}}} f_{i} w(\mathbf{v}) \mathrm{d} \Gamma
$$

where $\mathbf{D}$ is the linear elastic stiffness of the uncracked material, and $\boldsymbol{\varepsilon}$ the strain tensor. Substitution of (1) into (6) gives a discrete system of equations [12,13]. Since $k_{i}$ is a constant dependent on the opening of the cohesive crack, Equation (6) can be solved using a secant modulus iteration scheme when the length of the cohesive zone and external loads are given. The term secant modulus here refers to the fact that the stiffness matrix of XFEM obtained from (6) relates directly the total displacements to loads.

For a pure mode I problem, the first term of the asymptotic displacement field at the tip of a cohesive crack corresponds to a non-integer eigenvalue that gives a normal displacement discontinuity over the cohesive-crack faces. It has been derived in [23] and is

$$
\begin{aligned}
& u=\frac{r^{3 / 2}}{2 \mu} a_{1}\left[\left(\kappa+\frac{1}{2}\right) \cos \frac{3}{2} \theta-\frac{3}{2} \cos \frac{1}{2} \theta\right] \\
& v=\frac{r^{3 / 2}}{2 \mu} a_{1}\left[\left(\kappa-\frac{1}{2}\right) \sin \frac{3}{2} \theta-\frac{3}{2} \sin \frac{1}{2} \theta\right]
\end{aligned}
$$

where $\mu=E /[2(1+v)]$ is the shear modulus; the Kolosov constant is $\kappa=3-4 v$ for plane strain or $\kappa=(3-v) /(1+v)$ for plane stress; $E$ and $v$ are Young's modulus and Poisson's ratio, respectively. $(r, \theta)$ represent polar co-ordinates at the cohesive crack tip (Figure 1). Displacements (7) and (8) are used as the crack tip enrichment function in (1) for a mode I cohesive crack. The unknown coefficient $a_{1}$ depends on the softening law, and boundary and load conditions. It is considered as an additional degree of freedom at relevant enrichment nodes in XFEM. We mention in passing that in [23] complete asymptotic expansions for frictionless and frictional cohesive cracks have been obtained which are analogous to the Williams expansions in brittle solids. These expansions are valid for many commonly used separation laws, e.g. rectangular, linear, bilinear, exponential, etc with or without Coulomb friction.

In the numerical implementation of the XFEM for cohesive crack modelling, the integration over the domain in (6) is performed element by element using the quadrature rule in Xiao and Karihaloo [22] with a slight modification. We divide an element containing the crack tip into quadrilaterals whose boundaries align with the crack geometry as in [22]. However, we do not further subdivide the quadrilateral including the crack tip into four sub-quadrilaterals scaled down to the crack tip as in [22], since there is no singularity at a cohesive crack tip. An element cut by the crack but not containing the crack tip is divided into quadrilaterals whose boundaries align with the crack geometry. Fifteen order Gauss-Legendre quadrature is used for each quadrilateral of an element or the whole element containing node(s) enriched with branch functions (7) and (8). In the cohesive zone, the integration is performed by looping over all the segments. Three Gauss points are used on a segment with its length close to the element size for an accurate integration of the branch function. 
The flowchart for the simulation of the cohesive crack growth using an incremental-secant modulus iteration scheme is as follows:

Prescribe information on mesh and any traction-free crack, if it exists.

Carry out initial linear analysis to judge at which point $\sigma_{1}$ (recovered by, e.g.

SAR) first reaches $f_{t}$. The corresponding load is used as the initial load for the next iteration.

Loop over cohesive zone increments

A cohesive segment of given length (user defined) is added at the location (i.e. at the front of a pre-existing traction-free or cohesive crack) where the maximum tensile principal stress $\sigma_{1}$ (recovered by, e.g. SAR) reaches $f_{t}$. The initial guess opening for the first increment is set to be zero. Otherwise, the guess opening profile of the newly added cohesive segment is assumed to be of $c r^{3 / 2}$ type, where coefficient $c$ is determined by the opening displacement at the closest integration point of the previous cohesive crack segment.

The assumed load factor $\lambda^{1}$ for first load iteration is the converged value of the previous increment.

Iteration for balance load

Secant modulus iteration for solving the displacement field using (6); for a given external load and cohesive zone

If a linear softening law is used, the problem is linear and it is not necessary to check the convergence, exit iteration.

When a non-linear softening law is used, if the ratio of the increment of the crack opening displacement in the current iteration to the total opening displacement (we just consider the part whose opening is larger than 0.1 of the maximum opening) $\leqslant 10^{-4}$, the solution is deemed to have converged and exit secant modulus iteration.

\section{End secant modulus iteration}

The stresses at the tip of the cohesive crack are calculated using AVG or SAR.

If the maximum tensile principal stress $\sigma_{1}$ at the cohesive crack tip satisfies $\left|f^{(k-1)} / f_{t}\right|=\left\lfloor\left(\sigma_{1}-f_{t}\right) / f_{t}\right\rfloor<10^{-3}$, exit iteration for balance load. If $\left|f^{(k-1)} / f_{t}\right| \geqslant 10^{-3}$, compute the load factor of the next iteration step $(k)$ using a secant method:

$$
\begin{aligned}
& k=2: \lambda^{(2)}=\lambda^{(1)}\left(1.1-0.1 \sigma_{1} / f_{t}\right), \quad f^{(1)}=\sigma_{1}-f_{t} \\
& k>2: f^{(k-1)}=\sigma_{1}-f_{t} \\
& \lambda^{(k)}=\lambda^{(k-1)}-\left(\lambda^{(k-1)}-\lambda^{(k-2)}\right) f^{(k-1)} /\left(f^{(k-1)}-f^{(k-2)}\right) \\
& \text { If } \mid \frac{\lambda^{(k)}-\lambda^{(k-1)}}{\lambda^{(k-1)} \mid}>0.1, \lambda^{(k)}=\lambda^{(k-1)}\left(1+0.05 \frac{\lambda^{(k)}-\lambda^{(k-1)}}{\left|\lambda^{(k)}-\lambda^{(k-1)}\right|}\right)
\end{aligned}
$$

End iteration for balance load

If the last cohesive segment away from the tip has an opening at its central point $w>w_{c}$, it is removed from the cohesive zone and treated as a traction-free 
segment. The opening profile of the remaining part of the cohesive zone is used as the initial guess opening profile and the iterations for the new balance load are started for the modified cohesive zone.

If $w<w_{c}$, the balance load has been found for the current cohesive increment.

Output converged results.

If stop criterion has met, exit cohesive zone increment.

End loop over cohesive zone increments

\section{IMPROVEMENTS AND FURTHER STUDIES ON SAR}

\subsection{Elimination of possible ill-conditioning of the matrix}

MLS interpolants are widely used in the literature nowadays (see, e.g. [22, 25]) and thus not repeated here. In MLS smoothing of the stresses, the matrix may tend to become ill-conditioned (especially for a finer mesh) when higher-order monomial terms are used. This was overcome by using a larger domain of influence (DOI) including more sampling points in Xiao and Karihaloo [25]. An alternative way is to introduce locally normalized co-ordinates running from -1 to +1 as suggested by Zienkiewicz et al. [26]. This can be done as follows.

In the process of recovering the stress at a point with co-ordinates $\tilde{\mathbf{x}}$ using MLS, when we calculate the stress shape function of a sampling point with co-ordinates $\mathbf{x}_{I}$ in the DOI (for which the weight function $w\left(\tilde{\mathbf{x}}-\mathbf{x}_{I}\right)$ is non-zero), its co-ordinates $\mathbf{x}_{I}$ are normalized to

$$
\overline{\mathbf{x}}_{I}=\frac{\mathbf{x}_{I}-\tilde{\mathbf{x}}}{r_{\mathrm{doi}}}
$$

where $r_{\text {doi }}$ is the radius of the DOI. In calculating the MLS shape function for point $\tilde{\mathbf{x}}$, its normalized co-ordinates $\overline{\tilde{\mathbf{x}}}=0$ should be used instead of $\tilde{\mathbf{x}}$.

According to (11), the equation of a boundary

$$
n_{1} x+n_{2} y=p
$$

(where $\left(n_{1}, n_{2}\right)$ are the direction cosines of the unit outward normal to the boundary, and $p$ is a constant) will be changed into

$$
n_{1} \bar{x}+n_{2} \bar{y}=\frac{p-n_{1} \tilde{x}-n_{2} \tilde{y}}{r_{\text {doi }}}=\bar{p}
$$

\subsection{Influence of weight functions and sensitivity to the size of DOI}

We have tested four widely used weight functions. They are defined on $0 \leqslant d_{i} \leqslant r_{\text {doi }}$, or $0 \leqslant \bar{d}_{i}=$ $d_{i} / r_{\text {doi }} \leqslant 1$. When $\bar{d}_{i}>1$, all weight functions vanish. $d_{i}=\left|\tilde{x}-x_{i}\right|$ is the distance between the considered supporting point $x_{i}$ and the interpolation point $\tilde{x}$. These weight functions and their first and second derivatives are given below.

(I) Cubic polynomial:

$$
w_{C}\left(\bar{d}_{i}\right)=1-3 \bar{d}_{i}^{2}+2 \bar{d}_{i}^{3}
$$




$$
\begin{array}{cc}
\frac{\partial w_{C}\left(\bar{d}_{i}\right)}{\partial \bar{d}_{i}}=-6 \bar{d}_{i}+6 \bar{d}_{i}^{2}, & \left.\frac{\partial w_{C}\left(\bar{d}_{i}\right)}{\partial \bar{d}_{i}}\right|_{\bar{d}_{i}=1}=0 \\
\frac{\partial^{2} w_{C}\left(\bar{d}_{i}\right)}{\partial \bar{d}_{i}^{2}}=-6+12 \bar{d}_{i}, & \left.\frac{\partial^{2} w_{C}\left(\bar{d}_{i}\right)}{\partial \bar{d}_{i}^{2}}\right|_{\bar{d}_{i}=1}=6
\end{array}
$$

Equations (13) and (14) show that the application of the cubic weight function (12) leads to an exact $C^{1}$ continuous MLS interpolation function.

(II) Fourth-order spline function:

$$
\begin{gathered}
w_{S}\left(\bar{d}_{i}\right)=1-6 \bar{d}_{i}^{2}+8 \bar{d}_{i}^{3}-3 \bar{d}_{i}^{4} \\
\frac{\partial w_{S}\left(\bar{d}_{i}\right)}{\partial \bar{d}_{i}}=-12 \bar{d}_{i}+24 \bar{d}_{i}^{2}-12 \bar{d}_{i}^{3},\left.\quad \frac{\partial w_{S}\left(\bar{d}_{i}\right)}{\partial \bar{d}_{i}}\right|_{\bar{d}_{i}=1}=0 \\
\frac{\partial^{2} w_{S}\left(\bar{d}_{i}\right)}{\partial \bar{d}_{i}^{2}}=-12+48 \bar{d}_{i}-36 \bar{d}_{i}^{2},\left.\quad \frac{\partial^{2} w_{S}\left(\bar{d}_{i}\right)}{\partial \bar{d}_{i}^{2}}\right|_{\bar{d}_{i}=1}=0
\end{gathered}
$$

Equations (16) and (17) show that the application of the fourth order spline function (15) leads to an exact $C^{2}$ continuous MLS interpolation function.

(III) Gaussian exponential weight function:

$$
\begin{gathered}
w_{G}\left(\bar{d}_{i}\right)=\frac{\mathrm{e}^{-\left(\bar{d}_{i} / \alpha\right)^{2}}-\mathrm{e}^{-1 / \alpha^{2}}}{1-\mathrm{e}^{-1 / \alpha^{2}}}, \quad \alpha=0.4 \\
\frac{\partial w_{G}\left(\bar{d}_{i}\right)}{\partial \bar{d}_{i}}=-\frac{\mathrm{e}^{-\left(\bar{d}_{i} / \alpha\right)^{2}}}{1-\mathrm{e}^{-1 / \alpha^{2}}} \frac{2 \bar{d}_{i}}{\alpha^{2}},\left.\quad \frac{\partial w_{G}\left(\bar{d}_{i}\right)}{\partial \bar{d}_{i}}\right|_{\bar{d}_{i}=1}=-\frac{\mathrm{e}^{-1 / \alpha^{2}}}{1-\mathrm{e}^{-1 / \alpha^{2}}} \frac{2}{\alpha^{2}} \approx-0.024 \\
\frac{\partial^{2} w_{G}\left(\bar{d}_{i}\right)}{\partial \bar{d}_{i}^{2}}=-\frac{\mathrm{e}^{-\left(\bar{d}_{i} / \alpha\right)^{2}}}{1-\mathrm{e}^{-1 / \alpha^{2}}}\left(\frac{2}{\alpha^{2}}-\frac{4 \bar{d}_{i}^{2}}{\alpha^{4}}\right) \\
\left.\frac{\partial^{2} w_{G}\left(\bar{d}_{i}\right)}{\partial \bar{d}_{i}^{2}}\right|_{\bar{d}_{i}=1}=-\frac{\mathrm{e}^{-1 / \alpha^{2}}}{1-\mathrm{e}^{-1 / \alpha^{2}}}\left(\frac{2}{\alpha^{2}}-\frac{4}{\alpha^{4}}\right) \approx 0.278
\end{gathered}
$$

Equations (19) and (20) show that $C^{1}$ and $C^{2}$ continuity of the MLS interpolation function can only be reached approximately by using the Gaussian weight function (18).

(IV) Regularized weight function:

Most and Bucher [27] presented a regularized weight function for meshless shape functions to fulfil the interpolation conditions and the essential boundary conditions without any additional effort. Their interpolation gives much more stable results for the element-free Galerkin (EFG) method for varying size of the influence radius and for strongly distorted nodal arrangements than other weight functions. 
The weight function value of node $i$ at an interpolation point $\tilde{x}$ is

$$
w_{R}\left(\bar{d}_{i}\right)=w_{R i}(\tilde{x})=\frac{\left[\bar{d}_{i}^{2}+\varepsilon\right]^{-2}-(1+\varepsilon)^{-2}}{\varepsilon^{-2}-(1+\varepsilon)^{-2}}
$$

Assuming the regularization parameter $\varepsilon \ll 1$ (Most and Bucher [27] suggested the choice $\varepsilon=10^{-5}$ ) such that

$$
\frac{d_{\min }}{D} \gg \varepsilon^{1 / 2}
$$

where $d_{\min }$ is the minimal distance between two nodes, we have

$$
\begin{gathered}
\left|w_{R i}\left(x_{j}\right)-\delta_{i j}\right|_{\max } \approx\left[\left(\frac{d_{\min }}{D}\right)^{-4}-1\right] \varepsilon^{2} \\
\frac{\partial w_{R}\left(\bar{d}_{i}\right)}{\partial \bar{d}_{i}}=-\frac{4\left(\bar{d}_{i}^{2}+\varepsilon\right)^{-3} \bar{d}_{i}}{\varepsilon^{-2}-(1+\varepsilon)^{-2}},\left.\quad \frac{\partial w_{R}\left(\bar{d}_{i}\right)}{\partial \bar{d}_{i}}\right|_{\bar{d}_{i}=1} \\
=-\frac{4(1+\varepsilon)^{-3}}{\varepsilon^{-2}-(1+\varepsilon)^{-2}} \approx-4 \varepsilon^{2} \\
\frac{\partial^{2} w_{R}\left(\bar{d}_{i}\right)}{\partial \bar{d}_{i}^{2}}=-\frac{4\left(\bar{d}_{i}^{2}+\varepsilon\right)^{-3}}{\varepsilon^{-2}-(1+\varepsilon)^{-2}}+\frac{24\left(\bar{d}_{i}^{2}+\varepsilon\right)^{-4} \bar{d}_{i}^{2}}{\varepsilon^{-2}-(1+\varepsilon)^{-2}} \\
\frac{\partial^{2} w_{R}\left(\bar{d}_{i}\right)}{\left.\partial \bar{d}_{i}^{2}\right|_{\bar{d}_{i}=1}}=-\frac{4(1+\varepsilon)^{-3}}{\varepsilon^{-2}-(1+\varepsilon)^{-2}}+\frac{24(1+\varepsilon)^{-4}}{\varepsilon^{-2}-(1+\varepsilon)^{-2}} \approx 20 \varepsilon^{2}
\end{gathered}
$$

Equations (24) and (25) show that the application of the regularized weight function (21) leads to a nearly $C^{1}$ and $C^{2}$ continuous MLS interpolation function when $\varepsilon$ is small. Which of the above four weight functions gives the best results is discussed later in this section.

A finite plate with an inclined edge crack under uniaxial tension (ICLECT), as shown in Figure 3(a), is used for tests on the influence of the above weight functions on the stresses recovered by SAR. The geometrical parameters of the ICLECT considered here are: $c=0.6, w=h=1$, $\vartheta_{0}=30^{\circ}$. The conventional bilinear isoparametric Q4 elements are used as background elements. Plane stress conditions with unit thickness are assumed. All nodes on the bottom line $(y=-1)$ are fixed in the vertical ( $y$ ) direction, but just the extreme left point $(x=-0.5, y=-1)$ on the line is fixed also in the horizontal $(x)$ direction (i.e. the plate is subjected to uniaxial tension at both ends). Uniaxial tensile stress $\sigma_{y}=1$ is applied on the top boundary. Young's modulus $E=1.0$, and Poisson's ratio $v=0.25$. The ICLECT is divided into $80 \times 160$ square elements. Computational aspects are similar to those detailed in [22]. We will use the singular term in the Williams expansion but change its $r$ term from $r^{1 / 2}$ to $r^{3 / 2}$. Only one layer of nodes surrounding the crack tip is enriched. The quadrature rule in Xiao and Karihaloo [22] will be adopted in the following computations without modification. $2 \times 2$ Gauss-Legendre quadrature points are chosen as sampling points for SAR in all (enriched and un-enriched) elements. 


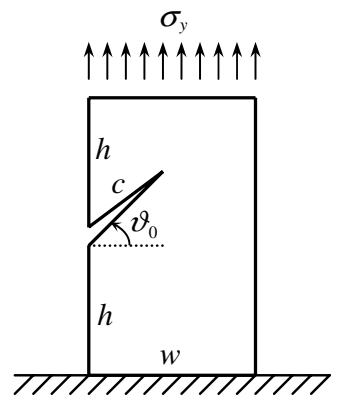

(a)

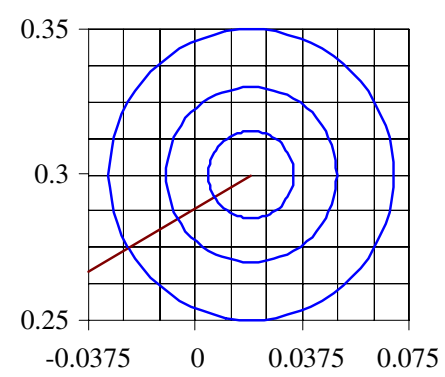

(b)

Figure 3. (a) A finite plate with an inclined edge crack under uniaxial tension (ICLECT); and (b) the mesh pattern at the crack tip and three circles with radii $r=0.015,0.03$ and 0.05 corresponding to a uniform division of $80 \times 160$ rectangular elements.

If the distance between a point and the crack is smaller than or equal to $1 / \sqrt{2}$ times the shorter diagonal of the element containing the point, and if the line perpendicular to the crack passing through this point crosses the crack, then a second order self-equilibrated stress field with six parameters meeting the local traction-free boundary conditions [25] is used, and the size of DOI is chosen as $\sqrt{2} r_{\text {doi }}$. Otherwise, a linearly self-equilibrated stress field with seven parameters meeting homogeneous equilibrium equations [25] is used, and the size of DOI is chosen as $r_{\text {doi }}$. Based on past experience [22] only two choices of $r_{\text {doi }}$ have been tested: $1 / \sqrt{2}$ times the shorter diagonal of the element containing the point for stress evaluation and length of the shorter diagonal of the element. For brevity, we will summarize the results instead of presenting them in graphs or tables to compare the efficiency of the adopted methods. The recovered stresses corresponding to the two different $r_{\text {doi }}$ are slightly different in the first two layers of elements surrounding the crack tip. The difference reduces further away from the crack tip, and almost disappears in the fourth layer of elements. For Gaussian exponential (18) and fourth order spline (15) weight functions, the stresses may exhibit spurious oscillations, especially for a small $r_{\text {doi }}$. For the regularized weight function (21), the sensitivity of the recovered stresses to $r_{\text {doi }}$ is much weaker than that of the other three weight functions when $\varepsilon \leqslant\left(d_{\min } / r_{\text {doi }}\right)^{2}$ (especially when $\left.\varepsilon \leqslant 0.1\left(d_{\min } / r_{\text {doi }}\right)^{2}\right)$. However, the spurious oscillations in the recovered stresses are more serious.

The weight function affects somewhat the recovered stresses. As expected, the difference between different weight functions reduces with the distance from the crack tip, and almost disappears in the fourth layer of elements. Overall, the cubic weight function (12) outperforms the rest. The recovered stresses using Gaussian weight function (18), the fourth-order spline function (15) and the regularized weight function (21) with $\varepsilon=\left(d_{\min } / r_{\mathrm{doi}}\right)^{2}$ are almost identical. The recovered stresses from the cubic weight function (12) are almost identical to those from the regularized weight function (21) with $\varepsilon=10\left(d_{\mathrm{min}} / r_{\mathrm{doi}}\right)^{2}$. No ill-conditioning was observed in the MLS process, if the local co-ordinates are normalized as in Section 3.1.

\subsection{Treatment of non-homogeneous boundary tractions}

The non-homogeneous traction conditions on the boundary (see Figure 4) can be treated by appending suitable special terms to the statically admissible stress fields meeting traction-free conditions on the boundary. If linearly distributed tractions are applied on the boundary segment $\mathrm{AB}$ of 


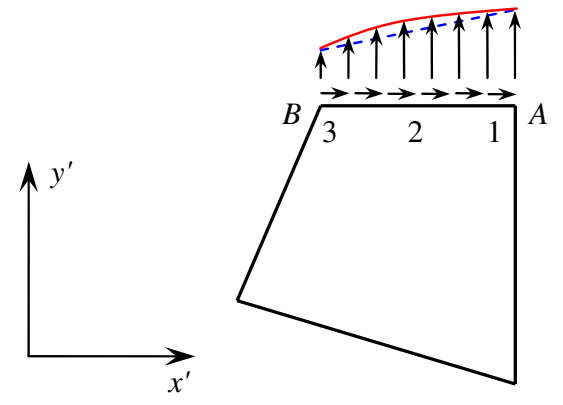

Figure 4. Linear and non-linear tractions on a boundary segment.

element ' $\mathrm{e}$ ', these tractions correspond to stresses at nodes $\mathrm{A}$ and $\mathrm{B}$ as $\left(\sigma_{y^{\prime} A}, \tau_{x^{\prime} y^{\prime} A}\right)$ and $\left(\sigma_{y^{\prime} B}\right.$, $\left.\tau_{x^{\prime} y^{\prime} B}\right)$ in the local co-ordinate system $x^{\prime} y^{\prime}$ with $x^{\prime}$-axis parallel with boundary AB and $y^{\prime}$-axis normal to it. The following self-equilibrated linear stress field in the local co-ordinate system can be used to enforce the tractions on the boundary:

$$
\begin{aligned}
\sigma_{x^{\prime}}^{0} & =0 \\
\sigma_{y^{\prime}}^{0} & =\beta_{2}+\beta_{5} x^{\prime}-\beta_{6} y^{\prime} \\
\tau_{x^{\prime} y^{\prime}}^{0} & =\beta_{3}+\beta_{6} x^{\prime}
\end{aligned}
$$

where

$$
\begin{aligned}
& \beta_{2}=\frac{\sigma_{y^{\prime} B} x_{A}^{\prime}-\sigma_{y^{\prime} A} x_{B}^{\prime}}{x_{A}^{\prime}-x_{B}^{\prime}}+\frac{\tau_{x^{\prime} y^{\prime} A}-\tau_{x^{\prime} y^{\prime} B}}{x_{A}^{\prime}-x_{B}^{\prime}} \\
& \beta_{3}=\frac{\tau_{x^{\prime} y^{\prime} B} x_{A}^{\prime}-\tau_{x^{\prime} y^{\prime} A} x_{B}^{\prime}}{x_{A}^{\prime}-x_{B}^{\prime}} \\
& \beta_{5}=\frac{\sigma_{y^{\prime} A}-\sigma_{y^{\prime} B}}{x_{A}^{\prime}-x_{B}^{\prime}} \\
& \beta_{6}=\frac{\tau_{x^{\prime} y^{\prime} A}-\tau_{x^{\prime} y^{\prime} B}}{x_{A}^{\prime}-x_{B}^{\prime}}
\end{aligned}
$$

If quadratically distributed tractions are applied on the boundary segment formed by the three nodes with co-ordinates $\left(x_{1}^{\prime}, y_{1}^{\prime}\right),\left(x_{2}^{\prime}, y_{2}^{\prime}\right),\left(x_{3}^{\prime}, y_{3}^{\prime}\right), y_{1}^{\prime}=y_{2}^{\prime}=y_{3}^{\prime}=y_{0}^{\prime}$, and given stresses $\left(\sigma_{y^{\prime} 1}\right.$, $\left.\tau_{x^{\prime} y^{\prime} 1}\right),\left(\sigma_{y^{\prime} 2}, \tau_{x^{\prime} y^{\prime} 2}\right),\left(\sigma_{y^{\prime} 3}, \tau_{x^{\prime} y^{\prime} 3}\right)$, the following self-equilibrated quadratic stress field in the local co-ordinate system can be used to enforce the tractions on the boundary:

$$
\begin{aligned}
\sigma_{x^{\prime}}^{0} & =0 \\
\sigma_{y^{\prime}}^{0} & =\beta_{2}+\beta_{5} x^{\prime}-\beta_{6} y^{\prime}+\beta_{9} x^{\prime 2}-2 \beta_{10} x^{\prime} y^{\prime} \\
\tau_{x^{\prime} y^{\prime}}^{0} & =\beta_{3}+\beta_{6} x^{\prime}+\beta_{10} x^{\prime 2}
\end{aligned}
$$


where

$$
\begin{aligned}
& \beta_{2}=\sigma_{y^{\prime} 2}-\beta_{5} x_{2}^{\prime}+\beta_{6} y_{0}^{\prime}-\beta_{9} x_{2}^{\prime 2}+2 \beta_{10} x_{2}^{\prime} y_{0}^{\prime} \\
& \beta_{3}=\tau_{x^{\prime} y^{\prime} 2}-\beta_{6} x_{2}^{\prime}-\beta_{10} x_{2}^{\prime 2} \\
& \beta_{5}=\frac{\sigma_{y^{\prime} 2}-\sigma_{y^{\prime} 1}}{x_{2}^{\prime}-x_{1}^{\prime}}-\beta_{9}\left(x_{2}^{\prime}+x_{1}^{\prime}\right)+2 \beta_{10} y_{0}^{\prime} \\
& \beta_{6}=\frac{\tau_{x^{\prime} y^{\prime} 2}-\tau_{x^{\prime} y^{\prime} 1}}{x_{2}^{\prime}-x_{1}^{\prime}}-\beta_{10}\left(x_{2}^{\prime}+x_{1}^{\prime}\right) \\
& \beta_{9}=\frac{1}{x_{3}^{\prime}-x_{1}^{\prime}}\left(\frac{\sigma_{y^{\prime} 3}-\sigma_{y^{\prime} 2}}{x_{3}^{\prime}-x_{2}^{\prime}}-\frac{\sigma_{y^{\prime} 2}-\sigma_{y^{\prime} 1}}{x_{2}^{\prime}-x_{1}^{\prime}}\right) \\
& \beta_{10}=\frac{1}{x_{3}^{\prime}-x_{1}^{\prime}}\left(\frac{\tau_{x^{\prime} y^{\prime} 3}-\tau_{x^{\prime} y^{\prime} 2}}{x_{3}^{\prime}-x_{2}^{\prime}}-\frac{\tau_{x^{\prime} y^{\prime} 2}-\tau_{x^{\prime} y^{\prime} 1}}{x_{2}^{\prime}-x_{1}^{\prime}}\right)
\end{aligned}
$$

This quadratic stress field meeting equilibrium and quadratic boundary traction conditions will be appended to the stress field meeting traction-free boundary conditions used in Section 3.2 in the SAR for simulations in the rest of this paper. The three points (not necessarily in the same element) on the boundary with the central one closest to the point where stresses are evaluated are used to construct the special stress field (27).

\section{NUMERICAL RESULTS}

In this section, we will analyse three typical mode I cohesive cracking problems of quasi-brittle materials using the incremental-secant modulus iteration scheme discussed in Section 2. These include a three point bend beam without any initial crack (Figure 5(a)) under linear and bilinear softening laws (we have actually assumed a very small initial crack of length $0.1 \mathrm{~mm}$ at the bottom midpoint of the beam), and an edge cracked plate under uniaxial tension (Figure 5(b)) with a bilinear softening law. A state of plane strain condition is considered for all specimens. The geometrical parameters for the three-point bend beam and the uniaxial tensile plate shown in Figure 5 are

$$
b=150 \mathrm{~mm}, \quad l=4 b, \quad t=b
$$

( $t$ is the specimen thickness in the out-of-plane direction). The initial edge crack in the tensile plate (Figure 5(b)) has a length of $a_{0}=15.1 \mathrm{~mm}$.

The beam and plate are discretized in the same way. Two meshes, as shown in Figure 6, are used in the analysis. The coarser mesh consists of $50 \times 100=5000$ rectangular elements, giving a total of 5151 nodes. The finer mesh consists of $150 \times 120=18000$ rectangular elements, giving a total of 18271 nodes. Both meshes are uniformly divided in $x$-direction. For the coarser mesh, the central 50 layers of elements have an identical height ( $y$-direction) of $3 \mathrm{~mm}$; the remaining elements have an identical height of $9 \mathrm{~mm}$. Therefore, elements in the central zone are $3 \times 3 \mathrm{~mm}^{2}$ squares. For the finer mesh, the central 60 layers of elements have an identical height of $1 \mathrm{~mm}$; the remaining elements have an identical height of $9 \mathrm{~mm}$. Therefore, elements in the central zone are 
(a)

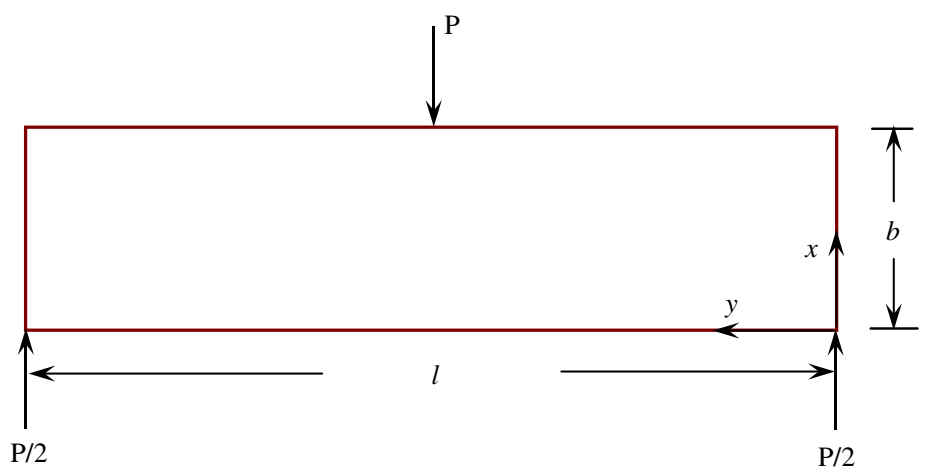

(b)

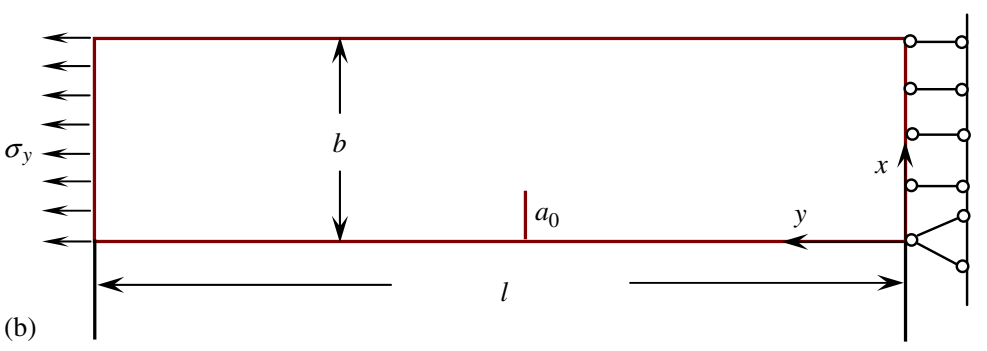

Figure 5. (a) An un-notched three-point bend beam (TPB); and (b) an edge-cracked plate under uniaxial tension.

$1 \times 1 \mathrm{~mm}^{2}$ squares. We will study global responses including the load-deformation behaviour and the evolution of the cohesive zone, as well as local properties including the opening profile and distribution of the cohesive stress in the cohesive zone, and displacements and stresses adjacent to the cohesive crack tip. More precisely, the displacements and stresses along the circle $r=3.5 \mathrm{~mm}$ (the second layer of elements in the coarser mesh, or the fourth layer of elements in the finer mesh) around the crack tip and along the line of extension of the crack are studied. The intention of using two meshes is to study the mesh size sensitivity of the global responses as well as of the crack tip fields. Furthermore, the results from the finer mesh will be used as a reference solution, since no analytical solutions are available and the stresses from this mesh along the circle $r=3.5 \mathrm{~mm}$ (the fourth layer of elements) obtained by direct differentiation of the displacements agree quite well with those obtained by the AVG and SAR, and can thus be regarded as accurate. The conventional 4-node bilinear isoparametric Q4 elements are also used as background elements. The first layer of nodes surrounding the cohesive crack tip (the elements that include the crack tip $k$ are defined as the first layer elements of the crack tip with enriched nodes; the nodes in the first layer elements are called the first layer enriched nodes) are enriched with (7) and (8). The potential fracture locus coincides with the specimen's axis of symmetry $y=300$ and thus the boundary of the mesh. However, in XFEM the crack is modelled by enriching the nodes on the crack faces with jump and branch functions without the double nodes that are used in the traditional FEM.

In the simulation, the first increment of the cohesive crack is $4.4 \mathrm{~mm}$, then the cohesive crack propagates by a segment of length $3 \mathrm{~mm}$ after each step in the coarser mesh, and by three segments of length $1 \mathrm{~mm}$ each in the finer mesh. 

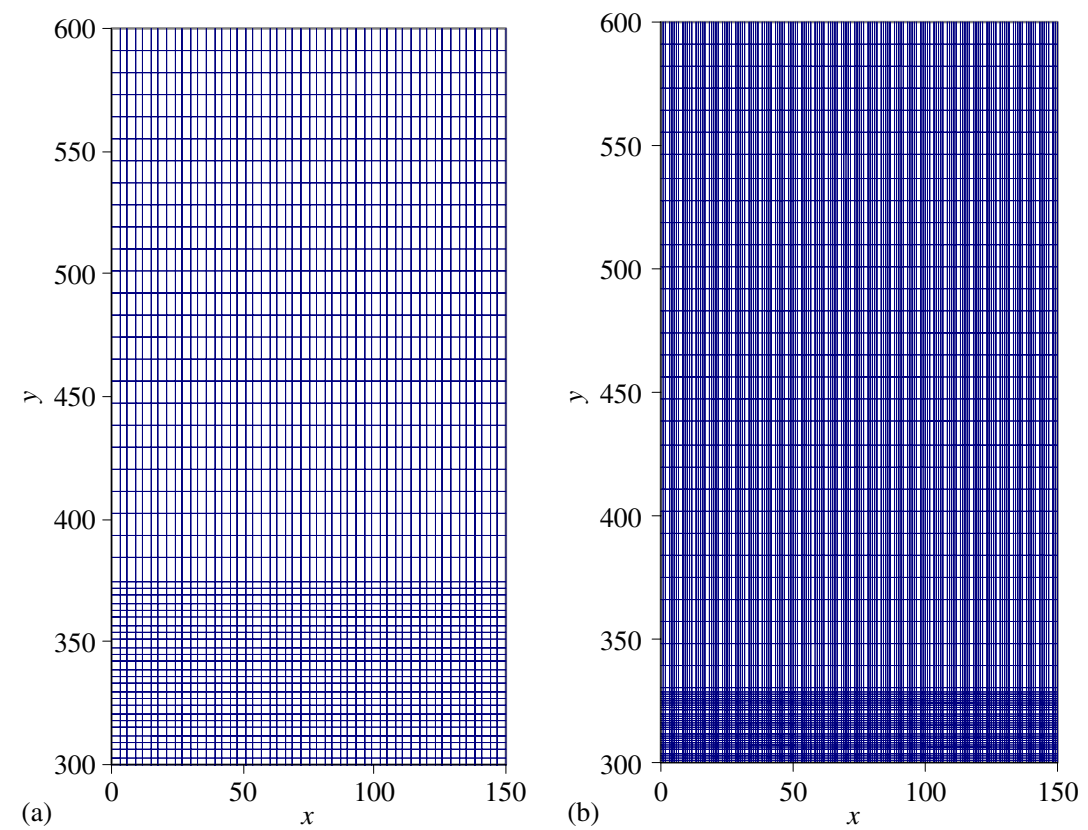

Figure 6. (a) Coarse; and (b) fine mesh for one half of the specimen to the left of mid-span (Figure 5).

Unless otherwise mentioned, the details of SAR are the same as in Section 3.2. The cubic polynomial weight function (12) is used. $r_{\text {doi }}$ is chosen as $1 / \sqrt{2}$ of the short diagonal of the element containing the point for stress evaluation. The stresses at the tip of the cohesive crack recovered by SAR are used to judge whether or not the tip will propagate.

For all examples studied below, the stresses for the finer mesh recovered by AVG and SAR are identical along the circle $r=3.5 \mathrm{~mm}$ and the line of extension of the crack. Therefore, all stress results are for the coarser mesh, unless mentioned otherwise.

The dimensions of the displacement and length parameters are in $\mathrm{mm}$, and those of the stresses in MPa.

\subsection{Three-point bend specimen with linear softening law}

The initiation and growth of a cohesive crack in such a beam has been studied extensively by Carpinteri and Colombo [28] using the FEM and the node release technique. The material properties with linear softening law (Figure 7) are

$$
E=36.5 \mathrm{GPa}, \quad v=0.1, \quad f_{t}=3.19 \mathrm{MPa}, \quad G_{F}=50 \mathrm{Nm}^{-1}
$$

where $E$ is Young's modulus, $v$ the Poisson's ratio, and $G_{F}$ the specific fracture energy, and

$$
\sigma_{y}=f_{t}\left(1-\frac{w}{w_{c}}\right)
$$




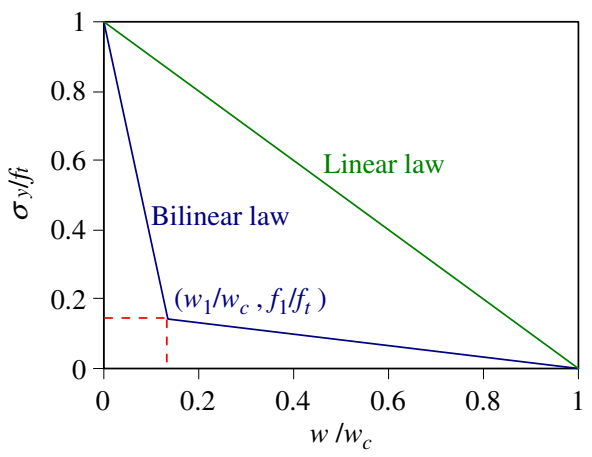

Figure 7. Linear and bilinear softening laws. The first and second branches of the bilinear diagram are referred to as (1) and (2) in Figures (14) and (19).

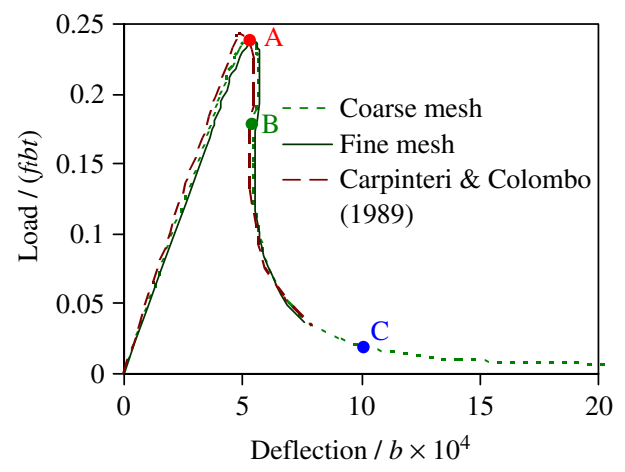

Figure 8. The non-dimensional load-midspan deflection curves of the three-point bend beam $\left(G_{F}=50 \mathrm{Nm}^{-1}\right)$.

Similar to Moës and Belytschko [13], $x$-direction of nodes with co-ordinates $(0,0)$ and $(0,600 \mathrm{~mm})$ and $y$-direction of the node with co-ordinates $(150,300 \mathrm{~mm})$ are constrained; the load is distributed over a length of $6 \mathrm{~mm}$ for the coarse mesh (Figure 6(a)) and $2 \mathrm{~mm}$ (two elements) for the fine mesh (Figure 6(b)). Since a low Poisson's ratio of 0.1 is used, the results are believed to be close to [28] where a plane stress condition is assumed and a concentrated load was considered.

The non-dimensional load-midspan deflection curves are shown in Figure 8. They are not sensitive to the mesh size and agree very well with the results of Carpinteri and Colombo [28] and Moës and Belytschko [13]. As the specific fracture energy is rather moderate $\left(G_{F}=50 \mathrm{Nm}^{-1}\right)$, the snap-back in the global load-deflection curve (Figure 8) is not pronounced, and the curve is not sensitive to the enrichment function [13] and the stress recovery technique used to determine the stresses at the cohesive crack tip. The evolution of the cohesive zone size as the cohesive tip travels through the beam shown in Figure 9, and the opening profile and distribution of cohesive 


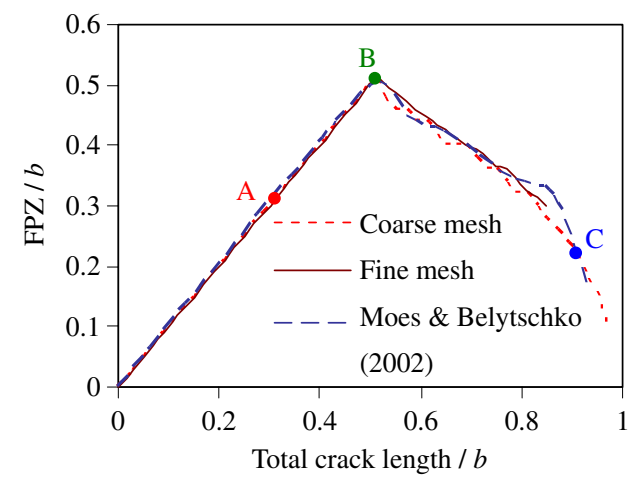

Figure 9. Evolution of the cohesive zone size as the cohesive tip travels through the beam.
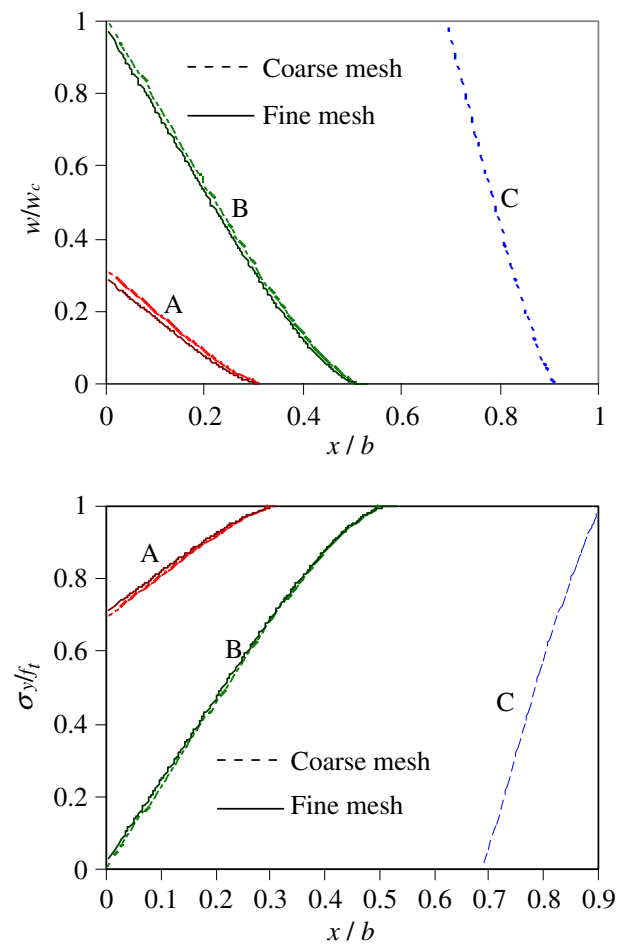

Figure 10. Opening profile and distribution of cohesive stresses in the FPZ at typical loading stages (see Figure 8).

stresses in the FPZ at several typical loading stages shown in Figure 10 are not sensitive to the mesh size. The evolution of the cohesive zone agrees very well with Moës and Belytschko [13]. Non-vanishing displacements and stresses along the circle $r=3.5 \mathrm{~mm}$, and the line of extension 

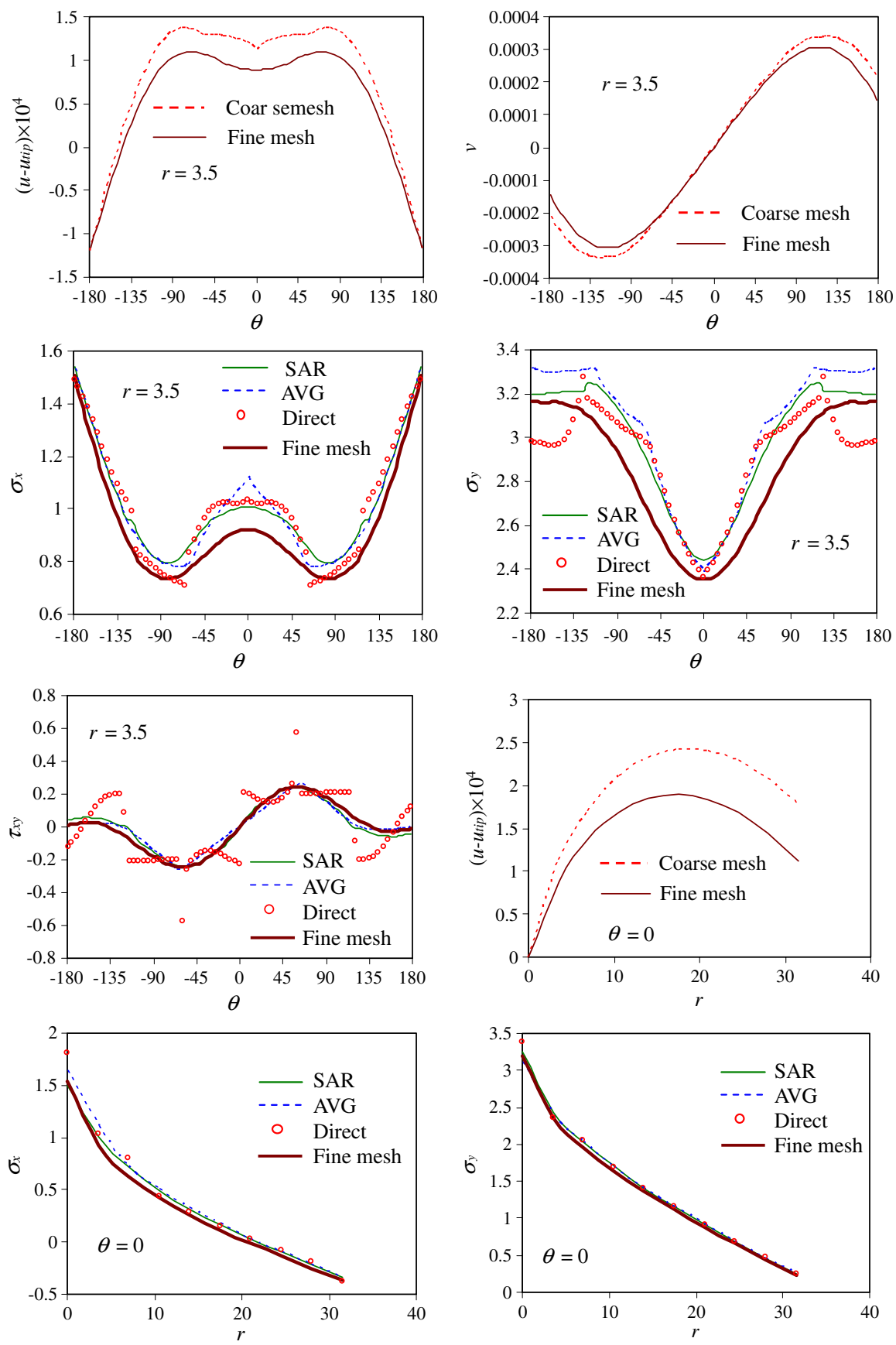

Figure 11. Non-vanishing displacements and stresses along the circle $r=3.5 \mathrm{~mm}$, and the line of extension of the crack at loading stage A (Figure 8). 

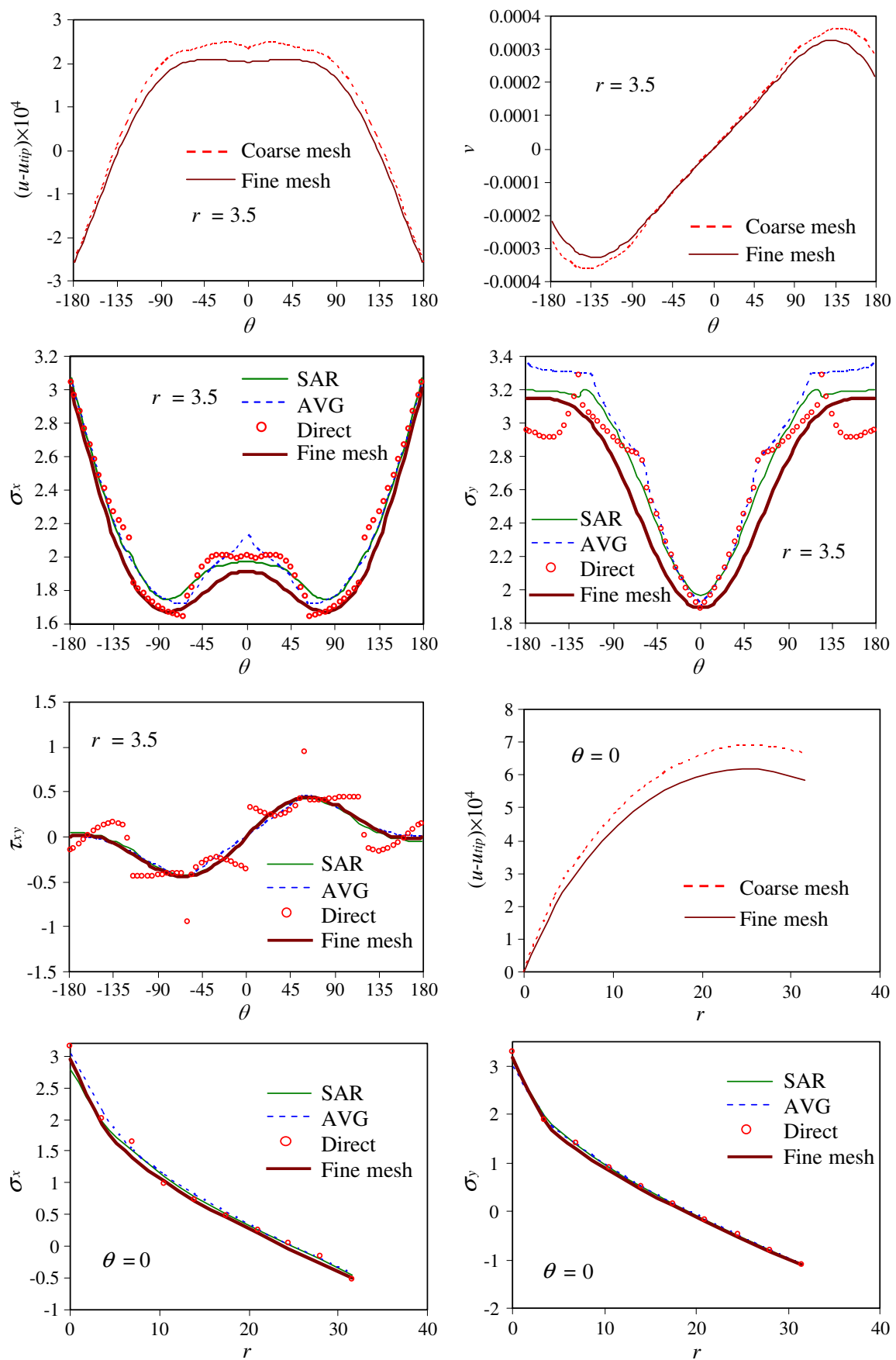

Figure 12. Non-vanishing displacements and stresses along the circle $r=3.5 \mathrm{~mm}$, and the line of extension of the crack at loading stage B (Figure 8). 


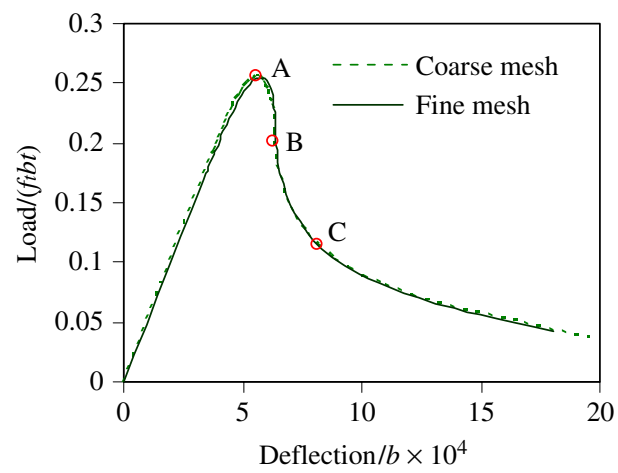

Figure 13. The non-dimensional load-midspan deflection curves of the three-point bend beam with a bilinear softening law.

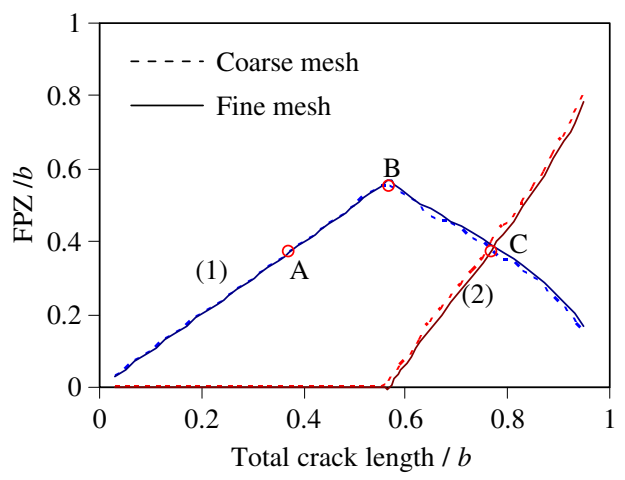

Figure 14. Evolution of the cohesive zone size as the cohesive tip travels through the beam. (1) and (2) correspond to the first and second branches of the bilinear softening diagram (Figure 7).

of the crack at loading stages A and B are shown in Figures 11 and 12, respectively. The basic characteristics of the results for both stages are generally similar. Both displacements and stresses on $r=3.5 \mathrm{~mm}$ are sensitive to the local mesh size. However, the stresses for the coarser mesh recovered by SAR are more accurate than direct differentiation and agree quite well with the finer mesh. Along the line of extension of the crack, the displacement $u$ in $x$-direction is quite sensitive to the local mesh size, but not the stresses. The SAR is more accurate than AVG close to the crack tip.

Note that close to the cohesive crack tip, the maximum values of the main stresses $\sigma_{x}$ and $\sigma_{y}$ occur on the cohesive crack face. This feature of the stress distribution for this type of specimen has also been noticed by Planas et al. [29].

The use of the enrichment function in the XFEM has been noticed to deteriorate the condition of the discrete system $[2,30]$. This does not affect global responses or the stresses and the strain 

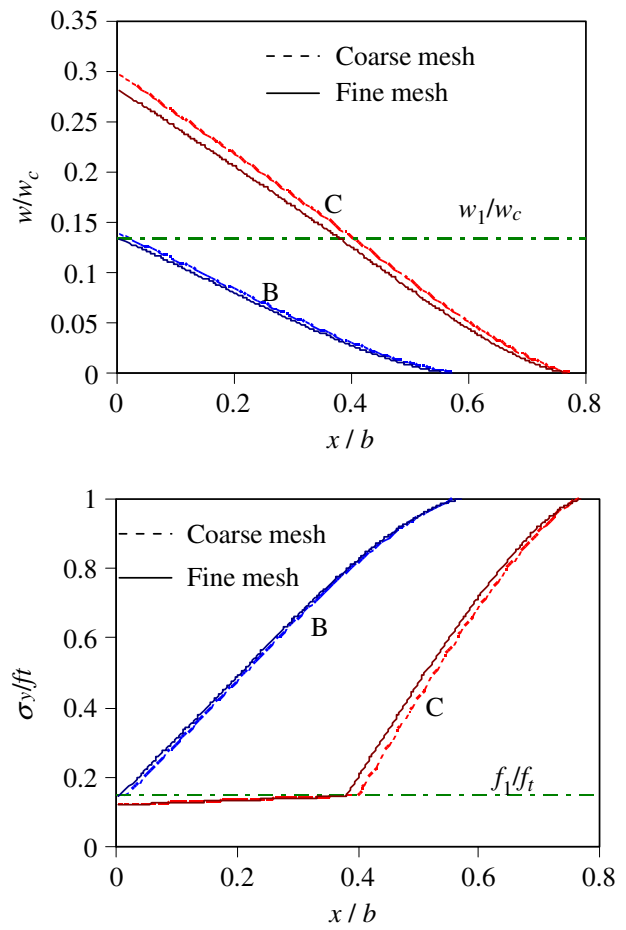

Figure 15. Opening profile and distribution of cohesive stresses in the FPZ at typical loading stages (Figure 13).

energy. However, it may affect significantly the displacements adjacent to the tip of the cohesive crack, so that a direct comparison of the coarse and fine mesh patterns (Figure 6) adopted in the analysis is not possible. Hence, we compare instead the displacements relative to the tip of the cohesive crack.

\subsection{Three point bend beam with bilinear softening law}

We now study the beam in Section 4.1 with the properties and a bilinear softening law obtained by Abdalla and Karihaloo [31] for a real normal strength concrete. The material properties are

$$
E=36.9 \mathrm{GPa}, \quad v=0.2
$$

and the bilinear softening law (Figure 7) is described by

$$
\sigma_{y}=\left\{\begin{array}{cl}
f_{t}\left[1-\frac{w\left(f_{t}-f_{1}\right)}{w_{1} f_{t}}\right], & 0 \leqslant w<w_{1} \\
\frac{f_{1} w_{c}}{\left(w_{c}-w_{1}\right)}\left(1-\frac{w}{w_{c}}\right), & w_{1} \leqslant w \leqslant w_{c}
\end{array}\right.
$$



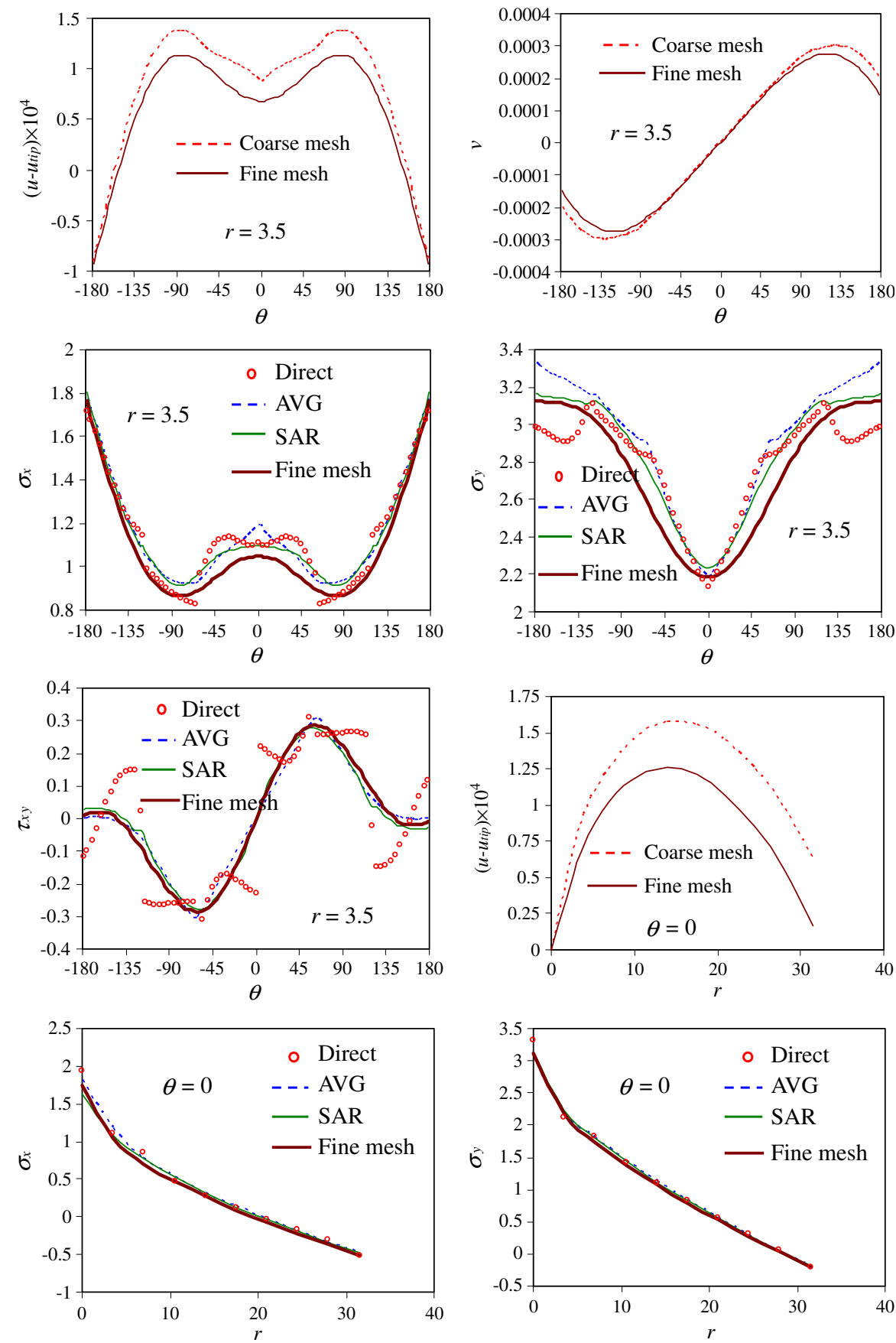

Figure 16. Non-vanishing displacements and stresses along the circle $r=3.5 \mathrm{~mm}$, and the line of extension of the crack at loading stage A (Figure 13). 

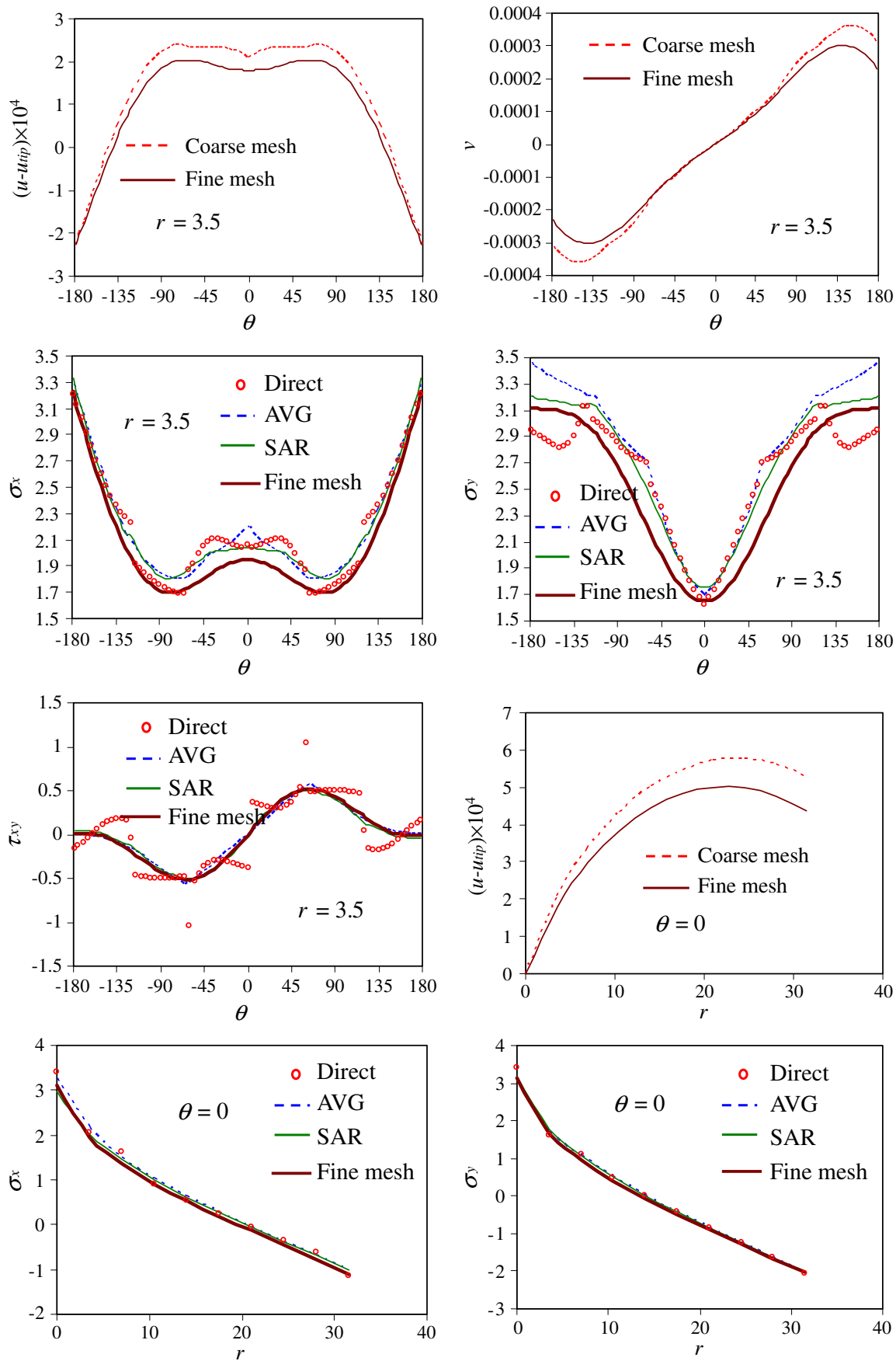

Figure 17. Non-vanishing displacements and stresses along the circle $r=3.5 \mathrm{~mm}$, and the line of extension of the crack at loading stage B (Figure 13). 


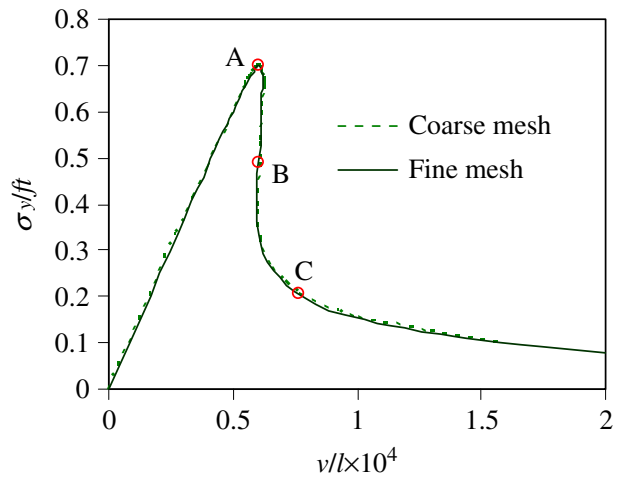

Figure 18. The non-dimensional tensile stress-central extension curves of the uniaxial single-edge notched tension plate.

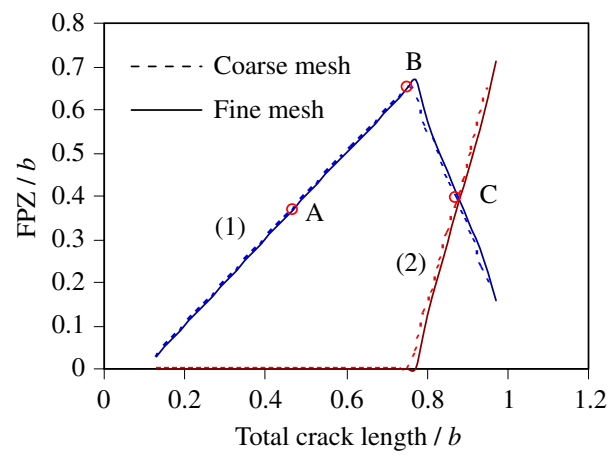

Figure 19. Evolution of the cohesive zone size as the cohesive tip travels through the plate. (1) and (2) correspond to the first and second branches of the bilinear softening diagram (Figure 7).

with

$$
\begin{aligned}
f_{t} & =3.14 \mathrm{MPa}, \quad f_{1}=0.455 \mathrm{MPa}, \quad w_{c}=0.279 \mathrm{~mm} \\
w_{1} & =0.0373 \mathrm{~mm}, \quad G_{F}=122 \mathrm{~N} / \mathrm{m}
\end{aligned}
$$

The applied constraints and loadings in the simulation are the same as in Section 4.1.

The non-dimensional load-midspan deflection curves are shown in Figure 13. The evolution of the cohesive zone size as the cohesive tip travels through the beam is shown in Figure 14. These global properties reveal very weak mesh size sensitivity. The opening profile and distribution of cohesive stresses in the FPZ at typical loading stages are shown in Figure 15. At loading stage B, these properties are almost independent of the mesh size. However, their mesh size dependence increases with the evolution of damage, as seen at loading stage C. Non-vanishing displacements and stresses along the circle $r=3.5 \mathrm{~mm}$, and the line of extension of the crack at loading stages A 

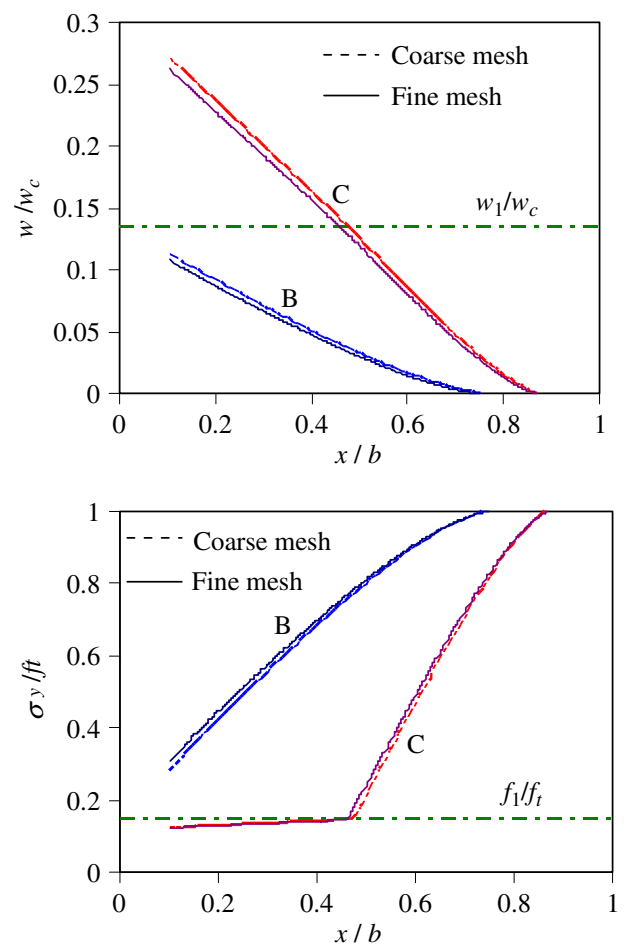

Figure 20. Opening profile and distribution of cohesive stresses in the FPZ at typical loading stages (Figure 18).

and B are shown in Figures 16 and 17, respectively. The basic characteristics of the results for both stages are generally similar. Both displacements and stresses on $r=3.5 \mathrm{~mm}$ are sensitive to the local mesh size. However, the stresses for the coarser mesh recovered by SAR are more accurate than direct differentiation and agree quite well with the finer mesh. Along the line of extension of the crack, the displacement $u$ in $x$-direction is quite sensitive to the local mesh size, but not the stresses. The SAR is more accurate than AVG close to the crack tip.

It is interesting to note that the angular distribution of displacements and stresses for linear (Figures 11 and 12) and bilinear laws (Figures 16 and 17) are quite similar.

\subsection{Edge-cracked uniaxial tensile plate with bilinear softening law}

Finally, we analyse an edge cracked plate under uniaxial tension shown in Figure 5(b). The material properties including the adopted bilinear softening law are the same as in Section 4.2.

The variation of the tensile stress $\sigma_{y}$ and displacement $v$ in $y$-direction at the central point of the loading edge is shown in Figure 18. Obviously, it shows no mesh dependency. The evolution of the size of the cohesive zone corresponding to the two branches of the bilinear softening law is shown in Figure 19. It also reveals very weak mesh dependence. The opening profile and distribution of cohesive stresses in the cohesive zone are shown in Figure 20, and show some weak mesh dependence. Non-vanishing displacements and stresses along the circle $r=3.5 \mathrm{~mm}$, and the line 

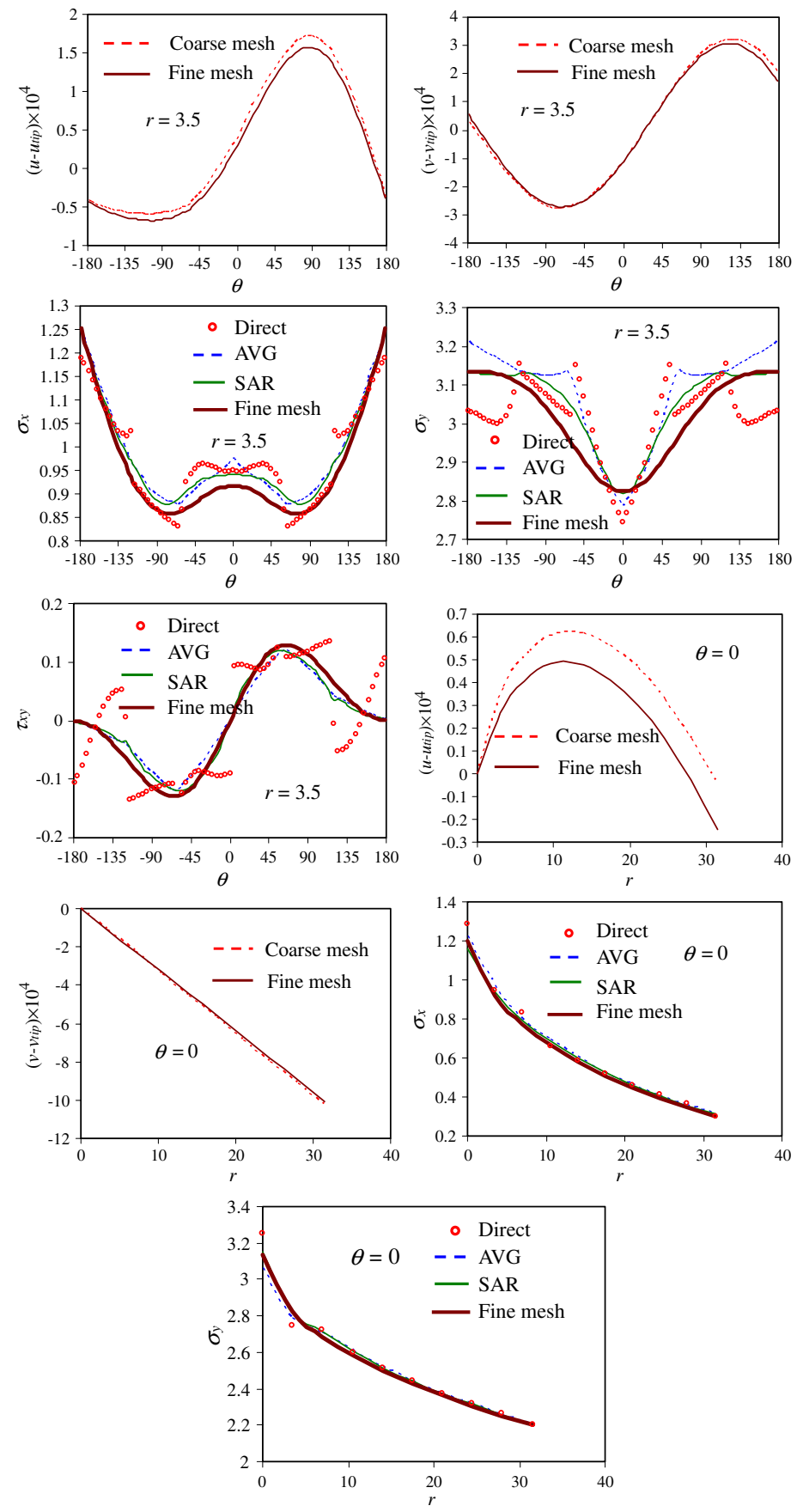

Figure 21. Non-vanishing displacements and stresses along the circle $r=3.5 \mathrm{~mm}$, and the line of extension of the crack at loading stage A (Figure 18). 

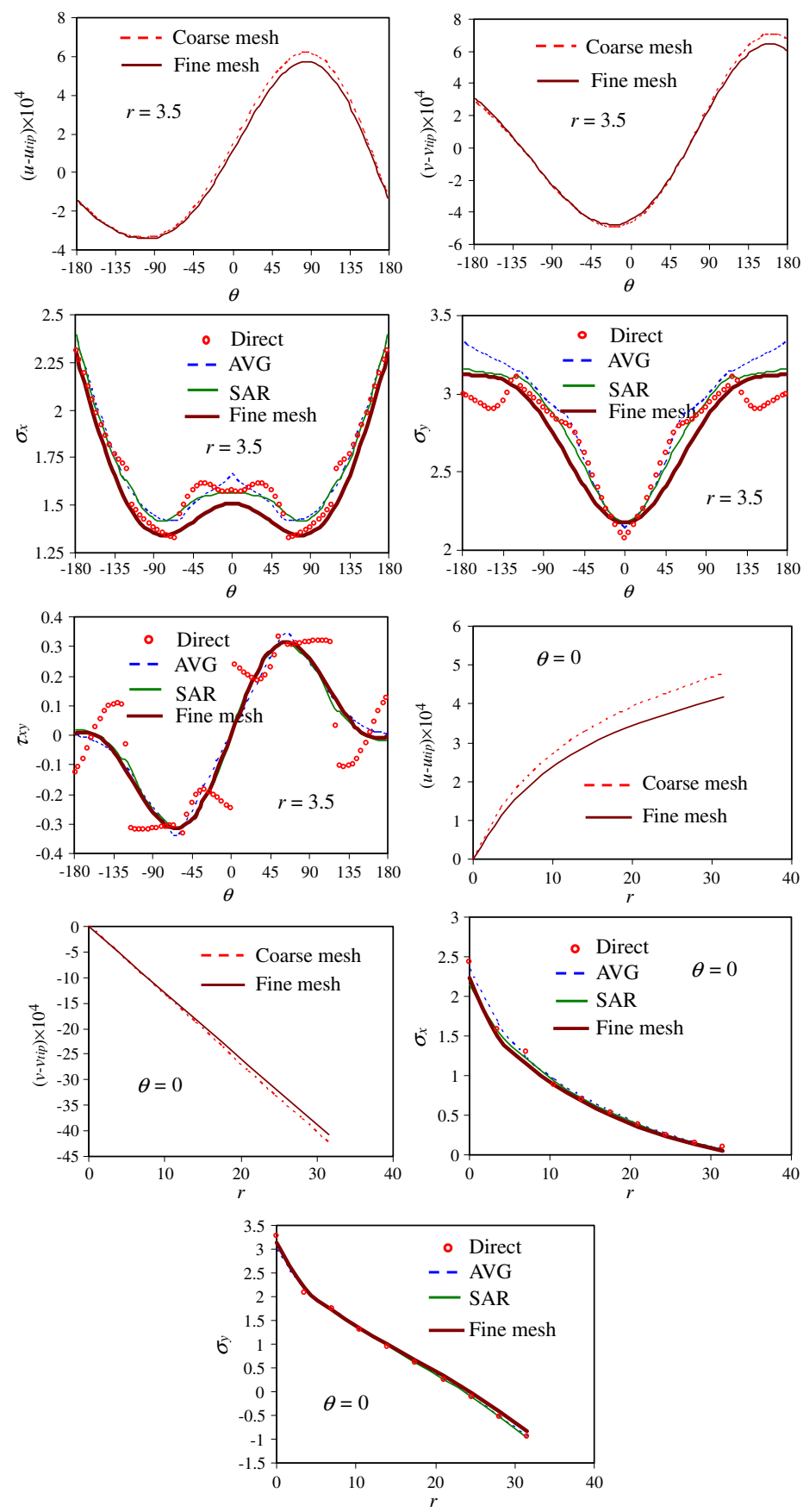

Figure 22. Non-vanishing displacements and stresses along the circle $r=3.5 \mathrm{~mm}$, and the line of extension of the crack at loading stage B (Figure 18). 
of extension of the crack at loading stages A and B are plotted in Figures 21 and 22, respectively. The basic characteristics of the results for both stages are generally similar. The displacements on $r=3.5 \mathrm{~mm}$ from the coarser mesh agree quite well with the finer mesh. The stresses for the coarser mesh obtained by SAR are more accurate than the direct differentiation and AVG, and agree quite well with the finer mesh. Along the line of extension of the crack, the displacement $u$ in $x$-direction is very sensitive to the mesh size, however the remaining non-vanishing displacement and stress components are not. The agreement between AVG, SAR and the finer mesh is generally very good, however, SAR is better than AVG close to the cohesive crack tip.

It is interesting to note that although the angular distribution of the displacement for the uniaxial tensile plate (Figures 21 and 22) is completely different from that of the three-point bend beam (Figures 11, 12, 16 and 17), the angular distributions of the stress are very similar.

\section{CONCLUSIONS AND DISCUSSION}

The normalization of the local co-ordinates makes the SAR more robust. The cubic weight function seems the most appropriate for SAR. Non-homogeneous tractions on the boundary can be handled by appending the proposed special stress fields.

The incremental-secant modulus iteration scheme is simple but powerful for the simulation of cracking or failure of quasi-brittle materials using the cohesive crack model with softening law composed of linear segments, e.g. the widely used linear and bilinear laws. It is stable, and converges in just a few iterations until the very last stage of failure. Although only mode I problems are studied, extension of the scheme to mixed mode problems with or without friction is straightforward. The corresponding enrichment functions (counterparts of (7) and (8) for mode I) can be found in [23].

Global responses like load-deformation curves, and evolution of the cohesive zone, are not sensitive to the size of the adopted mesh in the XFEM. However, local properties like the opening profile and distribution of the cohesive stresses in the cohesive zone, especially crack tip displacements and stresses are more sensitive to the mesh size. Although no singularity exists at the tip of a cohesive crack, the stresses obtained by direct differentiation of the displacements are not accurate, and cannot be used to predict accurately the growth of the tip. The SAR, which satisfies exactly the non-homogeneous cohesive tractions, gives more accurate results than the widely used AVG adjacent to the crack tip. Therefore, in the simulation of the failure process in quasi-brittle materials using a cohesive model together with the XFEM, if high accuracy of the local fields is required (e.g. these fields are used to judge the growth of the tip), a relatively fine mesh needs to be used together with accurate stress recovery methods, such as SAR. For this reason, the evolution of multiple cohesive cracks, as discussed in [14], should be treated with special care.

For mode I cohesive cracks, the stresses recovered by SAR are highly accurate along the line of extension of the cohesive crack, and can be used directly to predict the growth of the crack. For general mixed-mode cracks, further studies are required on whether the SAR stresses at the tip, or on a small circle enclosing a few (e.g. one or two) layers elements around the tip should be used to predict the growth of the crack.

\section{ACKNOWLEDGEMENTS}

Financial support from the European Commission KMM-Network of Excellence is gratefully acknowledged. 


\section{REFERENCES}

1. Moës N, Dolbow J, Belytschko T. A finite element method for crack growth without remeshing. International Journal for Numerical Methods in Engineering 1999; 46:131-150.

2. Strouboulis T, Copps K, Babuška I. The generalized finite element method. Computer Methods in Applied Mechanics and Engineering 2001; 190:4081-4193.

3. Karihaloo BL, Xiao QZ. Modelling of stationary and growing cracks in FE framework without remeshing: a state-of-the-art review. Computers and Structures 2003; 81:119-129.

4. Babuška I, Banerjee U, Osborn JE. Survey of meshless and generalized finite element methods: a unified approach. Acta Numerica 2003; 12:1-125.

5. Xiao QZ, Karihaloo BL. Recent developments of the extended/generalized FEM and a comparison with the FEM. In Developments and Applications of Solid Mechanics (Proceedings of the Symposium on Prof. M. G. Huang's 90th Birthday), Wu XP (ed). Press of University of Science and Technology of China: Hefei, China, 2005; 303-324.

6. Babuška I, Melenk JM. The partition of unity method. International Journal for Numerical Methods in Engineering 1997; 40:727-758.

7. Dugdale DS. Yielding of steel sheets containing slits. Journal of the Mechanics and Physics of Solids 1960; 8: 100-108.

8. Barenblatt GI. The mathematical theory of equilibrium of cracks in brittle fracture. Advances in Applied Mechanics 1962; 7:55-129.

9. Hillerborg A, Modeer E, Petersson PE. Analysis of crack formation and crack growth in concrete by means of fracture mechanics and finite elements. Cement and Concrete Research 1976; 6:773-781.

10. Elices M, Guinea GV, Gómez J, Planas J. The cohesive zone model: advantages, limitations and challenges. Engineering Fracture Mechanics 2002; 69:137-163.

11. Karihaloo BL. Fracture Mechanics and Structural Concrete. Addison-Wesley Longman: U.K., 1995.

12. Wells GN, Sluys LJ. A new method for modelling cohesive cracks using finite elements. International Journal for Numerical Methods in Engineering 2001; 50:2667-2682.

13. Moës N, Belytschko T. Extended finite element method for cohesive crack growth. Engineering Fracture Mechanics 2002; 69:813-833.

14. Remmers JJC, de Borst R, Needleman A. A cohesive segments method for the simulation of crack growth. Computational Mechanics 2003; 31:69-77.

15. de Borst R, Gutierrez MA, Wells GN, Remmers JJC, Askes H. Cohesive-zone models, higher-order continuum theories and reliability methods for computational failure analysis. International Journal for Numerical Methods in Engineering 2004; 60:289-315.

16. Hansbo A, Hansbo P. A finite element method for the simulation of strong and weak discontinuities in solid mechanics. Computer Methods in Applied Mechanics and Engineering 2004; 193:3523-3540.

17. Areias PMA, Belytschko T. A comment on the article 'A finite element method for simulation of strong and weak discontinuities in solid mechanics' by A. Hansbo, P. Hansbo. Computer Methods in Applied Mechanics and Engineering 2004; 193:3523-3540; Computer Methods in Applied Mechanics and Engineering 2006; 195: $1275-1276$.

18. Zi G, Belytschko T. New crack-tip elements for XFEM and applications to cohesive cracks. International Journal for Numerical Methods in Engineering 2003; 57:2221-2240.

19. Alfaiate J, Simone A, Sluys LJ. Non-homogeneous displacement jumps in strong embedded discontinuities. International Journal of Solids and Structures 2003; 40:5799-5817.

20. Mariani S, Perego U. Extended finite element method for quasi-brittle fracture. International Journal for Numerical Methods in Engineering 2003; 58:103-126.

21. Rubinstein AA. Computational aspects of crack path development simulation in materials with nonlinear process zone. International Journal of Fracture 2003; 119:L15-L20.

22. Xiao QZ, Karihaloo BL. Improving the accuracy of XFEM crack tip fields using higher order quadrature and statically admissible stress recovery. International Journal for Numerical Methods in Engineering 2006; 66: 1378-1410.

23. Xiao QZ, Karihaloo BL. Asymptotic fields at frictionless and frictional cohesive crack tips in quasi-brittle materials. Journal of the Mechanics of Materials and Structures 2006, in press.

24. Karihaloo BL, Abdalla HM, Xiao QZ. Deterministic size effect in the strength of cracked concrete structures. Cement and Concrete Research 2006; 36:171-188. 
25. Xiao QZ, Karihaloo BL. Statically admissible stress recovery using the moving least squares technique. In Progress in Computational Structures Technology, Topping BHV, Mota Soares CA (eds). Saxe-Coburg Publications: Stirling, Scotland, 2004; 111-138.

26. Zienkiewicz OC, Zhu JZ, Wu J. Superconvergent patch recovery techniques-some further tests. Communications in Numerical Methods in Engineering 1993; 9:251-258.

27. Most T, Bucher C. A moving least squares weighting function for the element-free Galerkin method which almost fulfills essential boundary conditions. Structural Engineering and Mechanics 2005; 21:315-332.

28. Carpinteri A, Colombo G. Numerical analysis of catastrophic softening behaviour (snap-back instability). Computers and Structures 1989; 31:607-636.

29. Planas J, Elices M, Guinea GV, Gómez FJ, Cendón DA, Arbilla I. Generalizations and specializations of cohesive crack models. Engineering Fracture Mechanics 2003; 70:1759-1776.

30. Béchet E, Minnebo H, Moës N, Burgardt B. Improved implementation and robustness study of the X-FEM method for stress analysis around cracks. International Journal for Numerical Methods in Engineering 2005; 64:1033-1056.

31. Abdalla HM, Karihaloo BL. A method for constructing the bilinear tension softening diagram of concrete corresponding to its true fracture energy. Magazine of Concrete Research 2004; 56:597-604. 